\title{
Characteristics of Tornado Events and Warnings in the Southeastern United States
}

\author{
AleXANDRA K. Anderson-Frey \\ Cooperative Institute for Mesoscale Meteorological Studies, University of Oklahoma, and NOAA/OAR \\ National Severe Storms Laboratory, Norman, Oklahoma \\ YVETTE P. RICHARDSON \\ The Pennsylvania State University, University Park, Pennsylvania
}

\author{
ANDREW R. DEAN, Richard L. ThOMPSON, AND BRYAN T. SMITH \\ Storm Prediction Center, Norman, Oklahoma
}

(Manuscript received 26 December 2018, in final form 29 May 2019)

\begin{abstract}
The southeastern United States has become a prime area of focus in tornado-related literature due, in part, to the abundance of tornadoes occurring in high-shear low-CAPE (HSLC) environments. Through this analysis of 4133 tornado events and 16429 tornado warnings in the southeastern United States, we find that tornadoes in the Southeast do indeed have, on average, higher shear and lower CAPE than tornadoes elsewhere in the contiguous United States (CONUS). We also examine tornado warning skill in the form of probability of detection (POD; percent of tornadoes receiving warning prior to tornado occurrence) and false alarm ratio (FAR; percent of tornado warnings for which no corresponding tornado is detected), and find that, on average, POD is better and FAR is worse for tornadoes in the Southeast than for the CONUS as a whole. These measures of warning skill remain consistent even when we consider only HSLC tornadoes. The Southeast also has nearly double the CONUS percentage of deadly tornadoes, with the highest percentage of these deadly tornadoes occurring during the spring, the winter, and around local sunset. On average, however, the tornadoes with the lowest POD also tend to be those that are weakest and least likely to be deadly; for the most part, the most dangerous storms are indeed being successfully warned.
\end{abstract}

\section{Introduction}

The common perception of tornadoes in the United States has historically been dominated by Tornado Alley, a region centered on the Great Plains in which tornadoes overwhelmingly occur in the late afternoon and early evening during springtime months (Kelly et al. 1978; Brooks et al. 2003; Coleman and Dixon 2014; Anderson-Frey et al. 2016, 2017; Krocak and Brooks 2018). In addition to this prominent Great Plains-centric discussion, the southeastern United States, nicknamed "Dixie Alley" (Schaefer et al. 1980), also has been identified as a region of particular concern when it comes to tornado risk. More focused research [such as the Verification of the Origins of Rotation in Tornadoes Experiment-Southeast (VORTEX-SE)] has emphasized the Southeast's unique juxtaposition of

Corresponding author: Alexandra K. Anderson-Frey, aka@ ualberta.ca physical risk factors (e.g., relatively high frequency of nocturnal tornadoes, disproportionate percentage of long-track tornadoes, low visibility of tornadoes in forested regions, a less easily defined storm season; Skaggs 1969; Kelly et al. 1978; Bounds and Soulé 1993; Egentowich et al. 2000; Boruff et al. 2003; Brooks et al. 2003; Coleman and Dixon 2014; Davis and Parker 2014; Sherburn and Parker 2014; Agee et al. 2016; AndersonFrey et al. 2016; Sherburn et al. 2016; Krocak and Brooks 2018; Long et al. 2018) and social risk factors (e.g., large segment of population in poverty, high population of elderly residents, high concentration of mobile homes; Grazulis 1990; Ashley 2007; Ashley et al. 2008; Sutter and Simmons 2010; Sherman-Morris 2013; Wallace et al. 2015; Childs and Schumacher 2018). Both of these have resulted in several prominent devastating tornado outbreaks in the region (e.g., Kincer 1936; Grazulis 1990; Egentowich et al. 2000; CDC 2012; May and Bigham 2012; Knupp et al. 2014) with, frequently, a disproportionately high loss of life versus comparable 
events in the Great Plains (Sims and Baumann 1972; Ashley 2007).

Much of the previous research into the unique characteristics of southeastern U.S. tornadoes has either focused on multidecadal summaries of nonenvironmental factors (seasonal and diurnal data, latitude, longitude, path width; e.g., Kelly et al. 1978; Grazulis 1990; Bounds and Soulé 1993; Boruff et al. 2003; Brooks et al. 2003; Coleman and Dixon 2014; Agee et al. 2016) or much shorter-term environmental studies (generally comprising fewer than five years of data; e.g., Davis and Parker 2014; Sherburn and Parker 2014; Sherburn et al. 2016), although Thompson et al. (2013) examined nine years of tornado near-storm environments in the context of the spatial distribution of convective mode.

Past studies have been centered on identifying and characterizing the physical attributes of southeastern tornadoes and their environments as inputs for ingredients-based forecasting and updates for conceptual models, with a special focus on the atypical tornadic environments common in this region (e.g., environments with marginal instability but large wind shear). Previous work, however, has not explicitly associated tornado warning skill with near-storm environmental parameters in this region. In addition to this forecast evaluation approach, this paper divides a relatively long-term tornado event, warning, and environment dataset by intensity, time of day, season, and storm mode, providing a wealth of directly comparable information that previously has been spread across several datasets and different times of record.

Defining the southeastern United States as in the following section, we make use of 4133 tornado events (refer to section 2 for the definition of an "event") and 16429 tornado warnings from 2003 to 2017. A 15-yr dataset helps partially offset the strong influence of individual tornado outbreaks when compared with a briefer period of study (although, for instance, the 27 April 2011 outbreak still features prominently in the data) without moving into multidecadal time scales that are more likely to encompass significant secular (nonmeteorological) shifts in the data. Associated with each event (or warning) is a set of model sounding parameters describing the environment in which the tornado occurred (or in which the warning was issued), and verification data are also available to help assess warning strengths and weaknesses in this high-vulnerability region of the country.

First, a description of the dataset and several relevant analytical methods are introduced. Southeastern tornado events and warnings will then be described on the basis of their near-storm environments, their seasonal and diurnal distributions, and finally their statistics pertaining to human vulnerability. Since tornado environments in the Southeast are disproportionately likely to have relatively low convective available potential energy (CAPE) and relatively high 0-6-km vector shear magnitude (Sherburn and Parker 2014; Sherburn et al. 2016), a subset of the data is created that consists of only these high-shear, low-CAPE events and warnings, and the properties of this subset are compared with the dataset as a whole. Finally, we discuss our findings and highlight future avenues of research suggested by the results.

\section{Data and methods}

The data used in this paper consist of an updated version of the dataset developed in Smith et al. (2012) and used in Anderson-Frey et al. (2016, 2017, 2018), consisting of all CONUS tornado events and warnings for 2003-17. Smith et al. (2012) discuss the creation of the tornado event dataset: county tornado segment data is filtered, and the highest $(\mathrm{E}) \mathrm{F}$-scale rating is kept within a given hour and $40 \times 40 \mathrm{~km}^{2}$ area; that is, multiple tornadoes occurring within a $40 \times 40 \mathrm{~km}^{2}$ grid box will be counted as a single event. Convective mode has been classified manually for each of these events through the examination of radar data and the use of a convective mode decision tree (Smith et al. 2012, their Fig. 2), resulting in mutually exclusive categories such as quasi-linear convective system (QLCS) and rightmoving supercell (RMS) as well as potentially overlapping categories such as tropical cyclone (TC). Only those tornado events or warnings occurring in Alabama, Arkansas, Georgia, Kentucky, Louisiana, Mississippi, and Tennessee are included so as to align with the area of interest for the VORTEX-SE campaign. Despite its location in the southeast of the country, Florida is omitted from this analysis, as it has a relatively high percentage of tornadoes associated with tropical cyclones and generally is not representative of the tornadic environments of the other states in the region (Edwards et al. 2012). Between 2003 and 2017, these 7 states (hereafter referred to as the Southeast) contained 4133 tornado events and 16429 tornado warnings.

Environmental data are matched with each tornado event using the grid box containing the initial tornado location and are matched with each tornado warning using the grid box containing the location of highest significant tornado parameter (STP; Thompson et al. 2003) within the warning area (Smith et al. 2012). The background for these mesoanalyses is obtained from the Rapid Update Cycle model (RUC; Benjamin et al. 2004) for January 2003-April 2012 or the Rapid Refresh model (RAP; Benjamin et al. 2016) for later 
a. Southeast Tornado Events (Time of Day, 2003-2017)

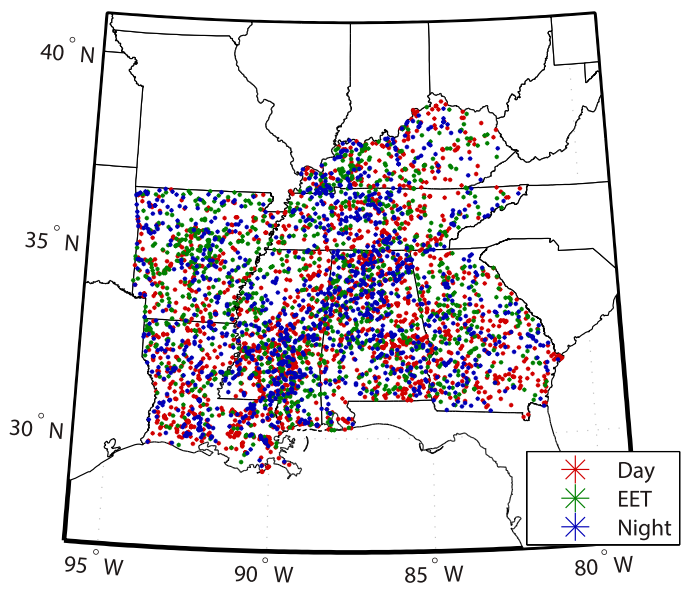

c. Southeast HSLC Tornado Events (Time of Day, 2003-2017)

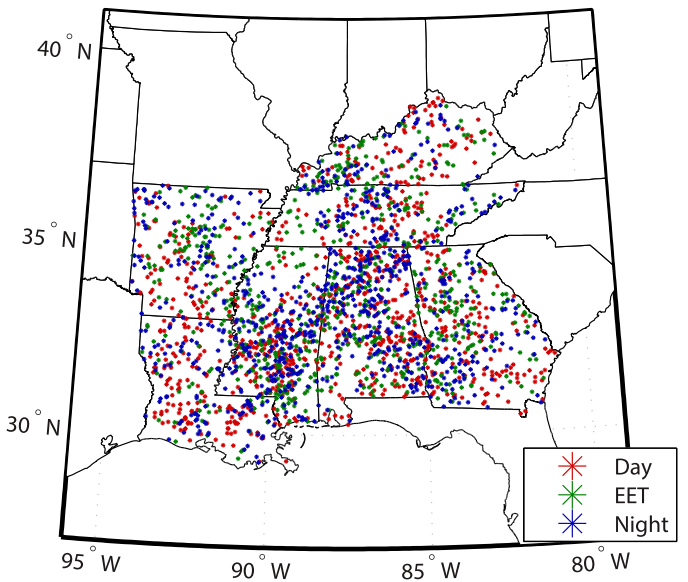

b. Southeast Tornado Events (Season, 2003-2017)

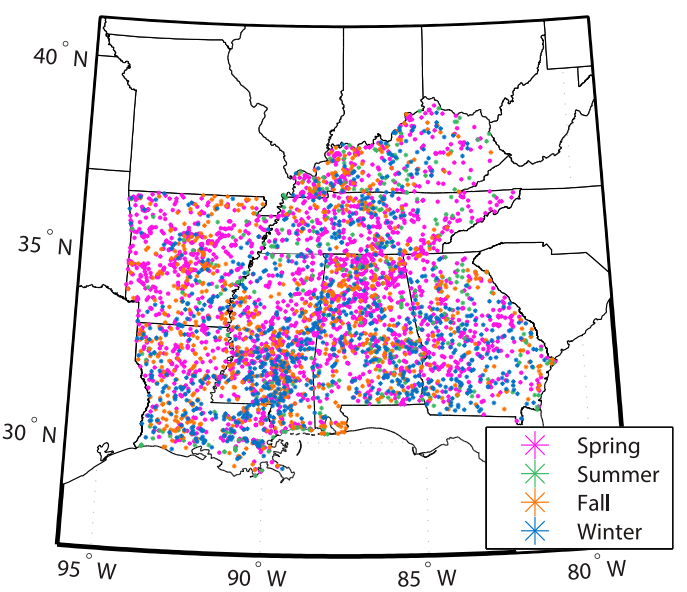

d. Southeast HSLC Tornado Events (Season, 2003-2017)

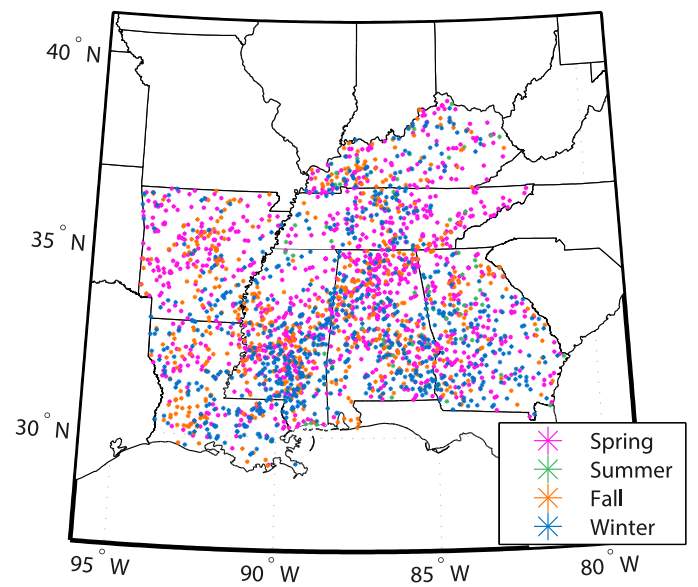

FIG. 1. Distribution of tornado events in the southeastern United States by (a),(c) time of day (day in red, EET in green, night in blue) and (b),(d) season (spring in pink, summer in green, fall in orange, winter in blue) for (top) all Southeast events and (bottom) Southeast HSLC events.

dates. Model horizontal grid spacing is $40 \mathrm{~km}$, and the models assimilate rawinsonde, profiler, radar, lightning, and other data (Benjamin et al. 2016, their Table 3). The reader is referred to Thompson et al. (2012) and Anderson-Frey et al. (2016) for a more indepth discussion of limitations of the dataset.

The STP (Thompson et al. 2003) is a parameter of interest that combines mixed-layer CAPE (MLCAPE), 0-6-km vector shear magnitude (SHR6), mixed-layer lifting condensation level (MLLCL), and 0-1-km stormrelative helicity (SRH1). ${ }^{1}$ Thompson et al. (2012) have

\footnotetext{
${ }^{1}$ Here, we make use of the fixed-layer STP (rather than the effective-layer STP) since effective-layer parameters are not available in the dataset until March 2005.
}

found that this parameter discriminates well between significantly severe but nontornadic supercell environments and significantly tornadic [i.e., capable of supporting tornadoes rated $(\mathrm{E}) \mathrm{F} 2+]$ supercell environments, and the individual parameter spaces of MLCAPE-SHR6 and MLLCL-SRH1 have been found to provide additional insight (Anderson-Frey et al. 2016, 2017). To avoid cluttered scatterplots in these two parameter spaces, kernel density estimation (KDE) is employed as a smoother, replacing each data point with a Gaussian kernel [see Anderson-Frey et al. (2016) for a more detailed explanation].

Figure 1 shows the geographical distribution of tornado events for the Southeast region. Here, time of day is split into three categories: day (from 12 to $2 \mathrm{~h}$ before local sunset), early evening transition (EET; from $2 \mathrm{~h}$ 
a. Tornado Event Environments

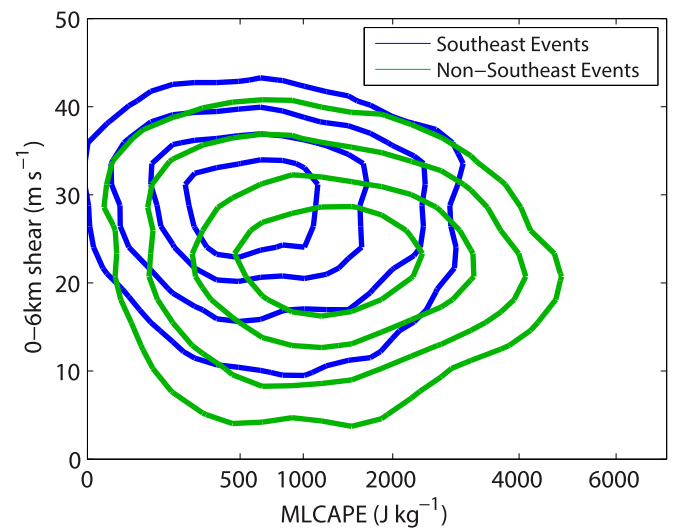

b. Tornado Event Environments

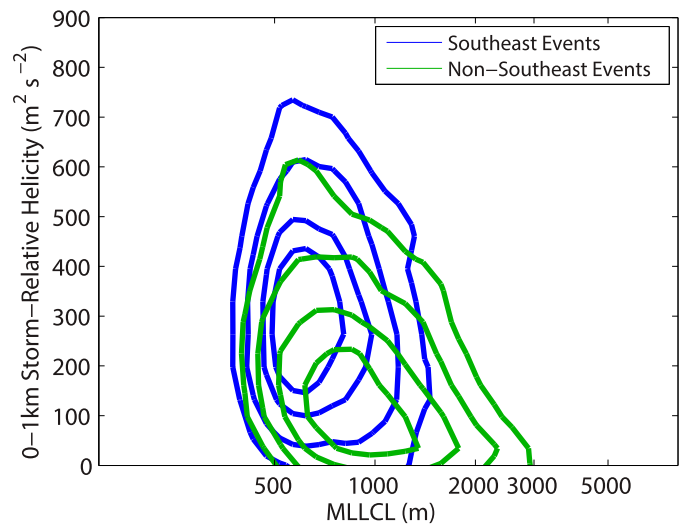

FIG. 2. Parameter-space distributions of only tornado events in the Southeast region (blue) and all CONUS nonSoutheast tornado events (green) for (a) the MLCAPE-SHR6 parameter space and (b) the MLLCL-SRH1 parameter space. Each set of contours is centered on the location of highest density of events, with the innermost contour enveloping $25 \%$ of the data, and contours moving outwards enveloping $50 \%, 75 \%$, and $90 \%$ of the data. Note that MLCAPE is on a nonlinear scale [converted to a speed via $\left(2 \times\right.$ MLCAPE $^{1 / 2}$ ], and MLLCL is on a logarithmic scale. A Gaussian kernel is used for the KDE.

before local sunset through $2 \mathrm{~h}$ after local sunset), and night (from 2 to $12 \mathrm{~h}$ after local sunset). Seasons are defined as follows: spring (MAM), summer (JJA), fall $(\mathrm{SON})$, and winter (DJF). Figures $1 \mathrm{a}$ and $1 \mathrm{~b}$ depict all Southeast tornado events, while Figs. 1c and 1d depict only those events with MLCAPE $\leq 1000 \mathrm{~J} \mathrm{~kg}^{-1}$ and SHR6 $\geq 18 \mathrm{~m} \mathrm{~s}^{-1}$ ( $\left.35 \mathrm{kt}\right)$, which constitutes the highshear, low-CAPE (HSLC) subset based on the definitions used by Sherburn and Parker (2014) and Sherburn et al. (2016).

Comparing time-of-day and seasonal distributions, it is apparent that nocturnal tornadoes (blue in Figs. 1a,c) occurred during these 15 years in similar swaths to the winter tornadoes (blue in Figs. 1b,d). Note also that, most apparently in western Arkansas, EET tornadoes (green in Figs. 1a,c) tend to occur in conjunction with springtime tornadoes (pink in Figs. 1b,d).

Comparing the distributions of all Southeast tornadoes with distributions of the HSLC subset of Southeast tornadoes, many daytime (red in Figs. 1a,c) and summer (green in Figs. 1b,d) tornadoes are eliminated from the HSLC subset, most prominently in western Tennessee and northern Mississippi. Intuitively, we might expect these tornadoes to have been eliminated on the basis of their greater mean CAPE and weaker mean shear (Anderson-Frey et al. 2017).

\section{Southeastern tornado events and warnings}

\section{a. Environmental factors}

Figure 2 draws a comparison between Southeast tornado environments and tornado environments for
non-Southeast events occurring in the rest of the contiguous United States (CONUS), supported by Thompson et al. (2013) using a similar period of record. In Fig. 2a, the distribution of events in which Southeast tornadoes occur has lower values of MLCAPE and higher values of SHR6 on average than the distribution of events in which tornadoes occur across other parts of the CONUS. Figure $2 b$ shows that Southeast tornadoes on average occur for higher values of SRH1 and somewhat lower MLLCL heights than tornadoes across other parts of the CONUS, which is a pattern that holds regardless of whether their parent storm morphologies are RMS or QLCS. All differences are statistically significant at the $p=0.05$ level using 10000-sample bootstrap confidence intervals.

This picture of the typical Southeast tornado as one occurring in high-shear but relatively moderate-buoyancy environments is one that should be qualified by season, by intensity, and by time of day, as there is a great deal of overlap between the Southeast and non-Southeast CONUS distributions. Figure 3 splits the Southeast tornadoes according to three different categories: $(\mathrm{E}) \mathrm{F}$ scale, season, and time of day (see section 2 for definitions of seasons and times of day).

Focusing on MLCAPE (Fig. 3a), MLCAPE values are generally similar for (E)F0-2 tornadoes, but tend to increase for more intense tornadoes, with very little overlap between (E)F2 and (E)F4+ tornadoes' boxes [note that, while all the (E)F5 tornadoes in this dataset occurred in conjunction with the 27 April 2011 outbreak, the (E)F5 boxplots here are consistent with those for tornadoes occurring across the entire CONUS (not shown)]. A similar signal was found for tornado events 
a. Southeast Tornado Event MLCAPE

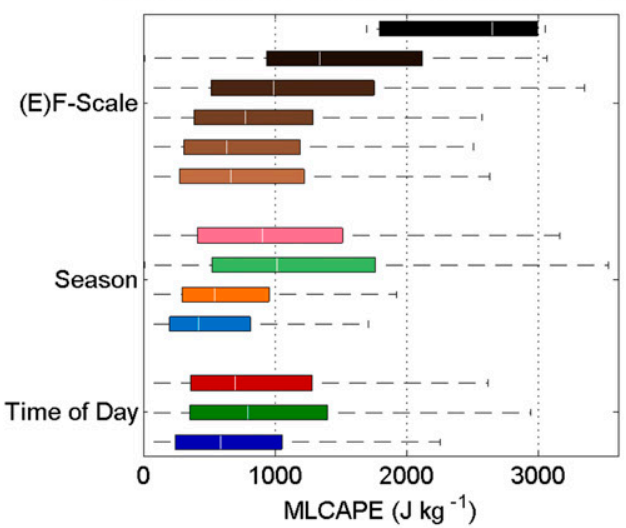

c. Southeast Tornado Event MLLCL

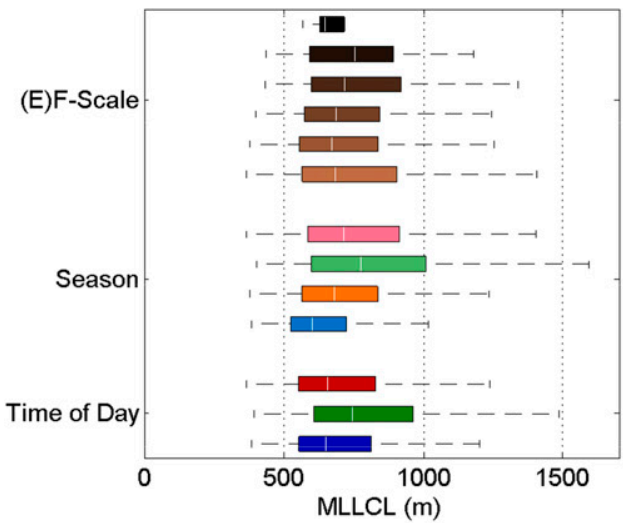

e. Southeast Tornado Event STP

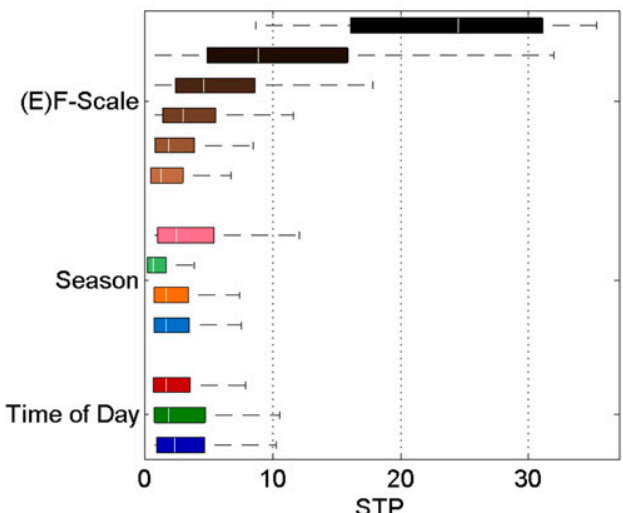

b. Southeast Tornado Event SHR6

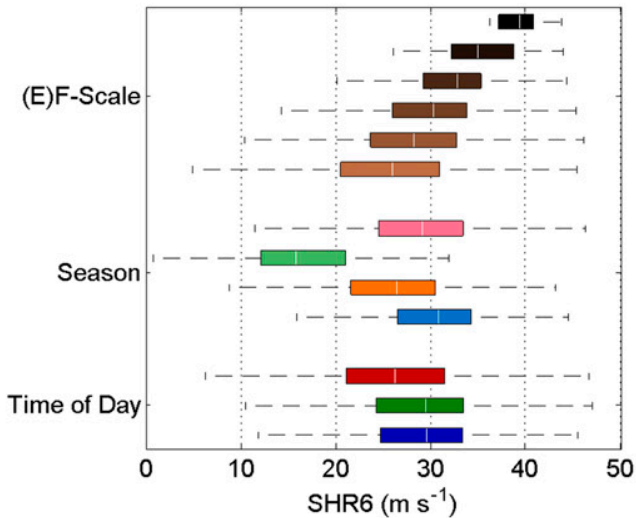

d. Southeast Tornado Event SRH1

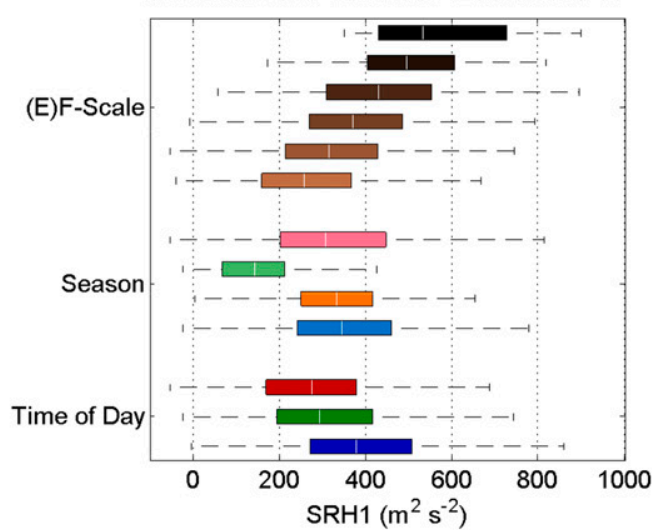

f. Legend

(E)F-Scale

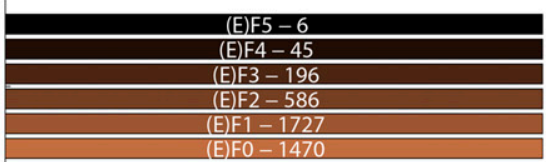

Season

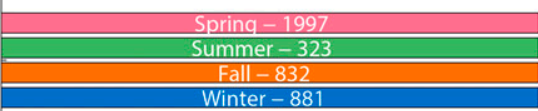

Time of Day

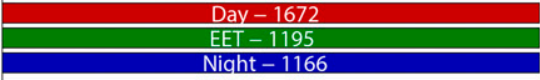

FIG. 3. Box-and-whiskers plots of (a) MLCAPE, (b) SHR6, (c) MLLCL, (d) SRH1, and (e) STP values for Southeast events. The white line inside each colored box indicates the median value for that category, the limits of the box are the 25th and 75th percentiles, and the whiskers extend to the most extreme data points that are not considered outliers (i.e., that lie within 1.5 times the interquartile range from the box). (f) A color bar legend, along with the number of Southeast events falling into each category.

for all convective modes by Smith et al. (2015) (cf. their Fig. 9). Somewhat higher MLCAPE values occur for the summer and near sunset, although there is a great deal of overlap in these distributions.

SHR6 (Fig. 3b) and SRH1 (Fig. 3d) share similar patterns, with values increasing more clearly with $(\mathrm{E}) \mathrm{F}$ scale (and the interquartile range of SHR6 tornadoes decreasing with (E)F scale), and minimum values occurring distinctly during the summer. On the other hand, MLLCL (Fig. 3c) shows a less distinct pattern, with heights in the 750-m range for all (E)F-scale ratings, and only marginally higher values during the summer and 
the EET. These low MLLCL heights correspond to relatively large values of low-level humidity, and hence result in more moderate outflow temperatures. Thus, as long as the outflow is not too cold (i.e., as long as MLLCL heights are not excessively high), the main difference seems to be in the dynamic lifting that comes from the low-level SRH, consistent with the findings of Coffer and Parker $(2015,2017)$.

Finally, Fig. 3e summarizes the results of the preceding four panels in the form of STP values. Despite MLLCL's lack of visible trend in (E)F scale, the other three parameters result in an STP value that clearly increases with $(\mathrm{E}) \mathrm{F}$-scale rating in a nonlinear way. Spring yields the highest STP values, due to favorable springtime values of all four STP component parameters. Summer's relatively low SHR6 and SRH1 and its somewhat higher MLLCL help offset its higher MLCAPE to result in the lowest STP values. Finally, time of day has virtually no STP pattern, with daytime tornadoes having only slightly lower mean STP (2.8) than EET or nocturnal tornadoes (3.3 and 3.4 , respectively).

Table 1 reproduces the values plotted in Fig. 3 but also depicts mean values by storm mode for RMS and QLCS tornadoes.

These distributions remain relatively consistent when examining only those Southeast tornadoes with an RMS storm mode (Table 1), with MLCAPE increasing from $846 \mathrm{~J} \mathrm{~kg}^{-1}$ for (E)F1 tornadoes to $2463 \mathrm{~J} \mathrm{~kg}^{-1}$ for (E)F5 tornadoes [note that all $(\mathrm{E}) \mathrm{F} 4+$ tornadoes in this dataset had the RMS storm mode]. For these RMS events, MLCAPE is only slightly larger in the summer $\left(1129 \mathrm{~J} \mathrm{~kg}^{-1}\right)$ than the spring $\left(1108 \mathrm{~J} \mathrm{~kg}^{-1}\right)$, and evening MLCAPE $\left(1005 \mathrm{~J} \mathrm{~kg}^{-1}\right)$ is only slightly larger than daytime MLCAPE $\left(934 \mathrm{~J} \mathrm{~kg}^{-1}\right)$. Hence, MLCAPE values in RMS tornadoes are much more closely tied to $(\mathrm{E}) \mathrm{F}$-scale value than to season or time of day

Similar to the full Southeast dataset, RMS tornadoes have a monotonic increase in SHR6 (SRH1) with (E)F scale, ranging from $26.1 \mathrm{~m} \mathrm{~s}^{-1}\left(287 \mathrm{~m}^{2} \mathrm{~s}^{-2}\right)$ at (E)F0 to $39.3 \mathrm{~m} \mathrm{~s}^{-1}\left(577 \mathrm{~m}^{2} \mathrm{~s}^{-2}\right)$ at (E)F5. The RMS subset also echoes the full Southeast dataset with maximum values of both parameters during the winter $\left(\right.$ SHR6 $\left.=30.4 \mathrm{~m} \mathrm{~s}^{-1}, \mathrm{SRH} 1=358 \mathrm{~m}^{2} \mathrm{~s}^{-2}\right)$ and minimum values during the summer (SHR6 $=18.6 \mathrm{~m} \mathrm{~s}^{-1}$, SRH1 $=184 \mathrm{~m}^{2} \mathrm{~s}^{-2}$ ). SHR6 is highest during the evening $\left(29.4 \mathrm{~m} \mathrm{~s}^{-1}\right)$ and SRH1 is highest overnight $\left(396 \mathrm{~m}^{2} \mathrm{~s}^{-2}\right)$. Thus, for RMS tornadoes, as for the entire Southeast dataset, intensity increases with the values of kinematic parameters, which have their smallest values for tornadoes occurring during the summer and daytime, and their largest values in the winter and near or after sunset.

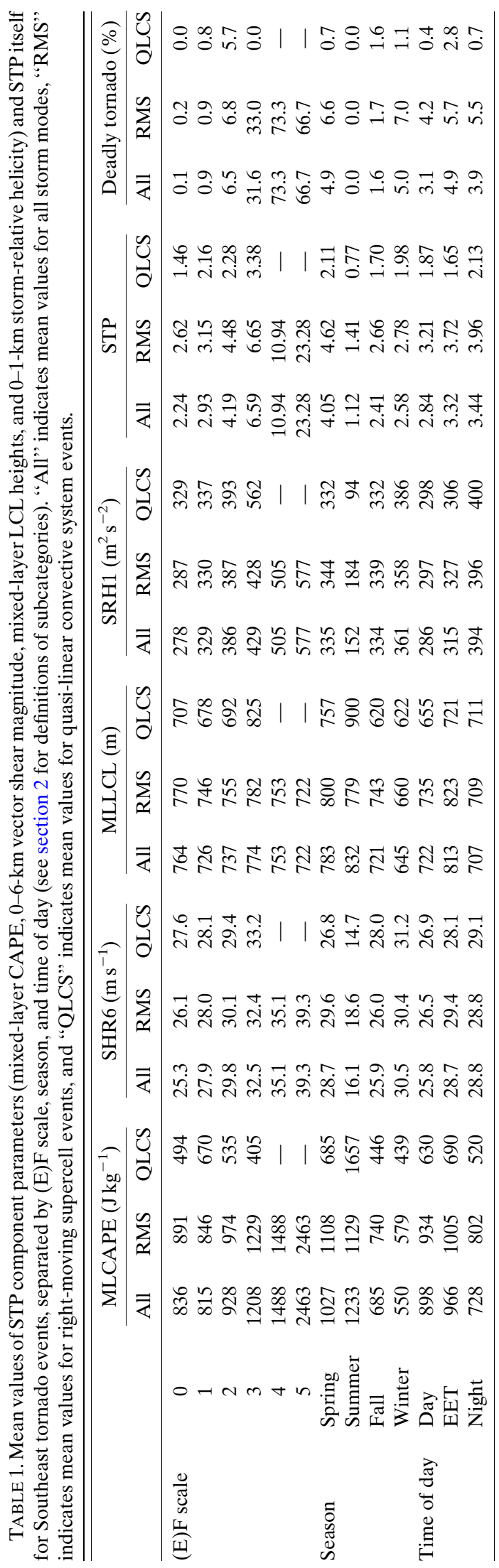


The RMS subset also has the full Southeast tornado dataset's lack of obvious trend in MLLCL heights with (E)F scale, but RMS MLLCL heights are somewhat higher in the spring $(800 \mathrm{~m})$ than the winter $(660 \mathrm{~m})$, and also higher in the evening $(823 \mathrm{~m})$ than at night $(709 \mathrm{~m})$. Overall, the RMS subset follows the same STP behavior as for the full dataset; STP increases with (E)F scale [from 2.62 at (E)F0 to 23.28 at (E)F5], and is highest for springtime (4.62) and overnight (3.96) events.

In contrast to RMS tornadoes and the Southeast dataset as a whole, QLCS tornadoes (Table 1) do not have a linear increase in MLCAPE with (E)F scale, with (E)F0-3 mean values of 494, 670, 535, and $405 \mathrm{~J} \mathrm{~kg}^{-1}$, respectively. MLCAPE is considerably higher in the summer for QLCS events $\left(1657 \mathrm{~J} \mathrm{~kg}^{-1}\right)$ than in the spring $\left(685 \mathrm{~J} \mathrm{~kg}^{-1}\right)$. Finally, similar to RMS events and the Southeast dataset as a whole, evening MLCAPE for QLCS events $\left(690 \mathrm{~J} \mathrm{~kg}^{-1}\right)$ is again somewhat higher than daytime MLCAPE for QLCS events $\left(630 \mathrm{~J} \mathrm{~kg}^{-1}\right)$. The linear increase in SHR6 and SRH1 with $(\mathrm{E}) \mathrm{F}$ scale is also apparent for QLCS tornadoes, with SHR6 (SRH1) increasing from $27.6 \mathrm{~m} \mathrm{~s}^{-1}\left(329 \mathrm{~m}^{2} \mathrm{~s}^{-2}\right)$ at (E)F0 to $33.2 \mathrm{~m} \mathrm{~s}^{-1}\left(562 \mathrm{~m}^{2} \mathrm{~s}^{-2}\right)$ at (E)F3. Also in agreement with RMS tornadoes and the full Southeast tornado dataset, QLCS values of both measures of shear are highest for winter tornadoes $\left(\right.$ SHR6 $=31.2 \mathrm{~m} \mathrm{~s}^{-1}$, SRH1 = $386 \mathrm{~m}^{2} \mathrm{~s}^{-2}$ ) and lowest for tornadoes occurring during the summer $\left(\right.$ SHR6 $=14.7 \mathrm{~m} \mathrm{~s}^{-1}$, SRH1 $=94 \mathrm{~m}^{2} \mathrm{~s}^{-2}$ ).

Similar to the lack of obvious trend in MLLCL heights with (E)F scale for all Southeast events and the RMS subset, the MLLCL heights of QLCS tornadoes for (E)F0-3 intensities are 707, 678, 692, and $825 \mathrm{~m}$, respectively. QLCS MLLCL values are considerably higher for the summer $(900 \mathrm{~m})$ than for the spring $(757 \mathrm{~m})$, fall $(620 \mathrm{~m})$, or winter $(622 \mathrm{~m})$ months, and are only marginally higher during the EET $(721 \mathrm{~m})$ than the daytime $(655 \mathrm{~m})$ or overnight $(711 \mathrm{~m})$. Overall, similar to the patterns for all Southeast events and for the RMS subset, the STP for QLCS events increases with (E)F scale from 1.46 to 3.38, is highest for spring (2.11) and lowest for summer (0.77). In contrast to the results for RMS tornadoes, however, QLCS tornadoes have their lowest STP during the evening (1.65), not during the day (1.87). A more striking difference between RMS and QLCS tornadoes, however, is that the mean (E)F-scale values of QLCS tornadoes do not increase with MLCAPE in the way that RMS tornadoes' (E)F-scale values do; the kinematic environment is more relevant than the thermodynamic environment in determining the intensity of these QLCS tornadoes.

The plots in Fig. 4 depict the shift in various relevant atmospheric parameters depending on the start time of a given tornado relative to local sunset; each season is depicted in a different color, and each tornado event's start time has been binned into its rounded-down hour. Note that summer events are considerably less common than the others (324 events, vs 854 in fall as the nextsmallest seasonal category), resulting in a noisier plot; see Fig. $4 \mathrm{f}$ for the number of events in each hourly bin.

Unsurprisingly, MLCAPE (Fig. 4a) is generally highest for tornadoes occurring in the spring and summer, and it peaks in spring and fall for tornadoes occurring around local sunset, at the warmest time of day. Both SHR6 (Fig. 4b) and SRH1 (Fig. 4d) are considerably lower for tornadoes occurring in the summer than in any other season, and SRH1 in particular peaks for all seasons when tornadoes occur overnight. MLLCL (Fig. 4c) has more of a diurnal trend for tornadoes occurring in summer and spring than in fall or winter, with somewhat higher MLLCL heights for late-afternoon or early evening tornadoes.

Outside of springtime, the summary plot of STP (Fig. 4e) shows little coherent pattern (possibly a little higher in the evening than overnight; the postsunset peak in summer is striking but is likely an artifact of the relatively small dataset for that season). Spring, however, has a coherent and well-defined pattern of values, going from a peak hourly mean of about 4.2 for tornadoes occurring around sunset to an hourly mean of about 2.8 for tornadoes occurring around sunrise.

Tornadoes in the Southeast are significant [i.e., rated (E)F2+] about $21.0 \%$ of the time; this, too, has a seasonal and diurnal variation (Table 2). Summertime Southeast tornadoes are significant only $7.6 \%$ of the time, and fall tornadoes only $13.7 \%$ of the time, whereas spring and winter tornadoes are much more commonly significant $(24.9 \%$ and $24.6 \%$, respectively). A disproportionately high percentage of nocturnal tornadoes are significant $(26.0 \%$, vs $20.4 \%$ of EET tornadoes and $17.1 \%$ of daytime tornadoes) in the Southeast. This may, perhaps, be the result of a reporting bias wherein tornadoes are simply less likely to be reported overnight unless they cause substantial damage, with weaker tornado damage attributed to straight-line winds if it is observed at all.

(E)F0 tornadoes in particular are much more common during the day ( $47.4 \%)$ than during the EET $(30.6 \%)$ or especially during the night $(22.0 \%)$, and the potential for population biases in the (E)F0 dataset is a topic of open discussion in the literature (e.g., Agee and Childs 2014). In contrast, even (E)F1 tornadoes show a closer balance, with $39.8 \%$ during the day, $29.0 \%$ during the EET, and $31.2 \%$ at night. On the other hand, meteorology may be the strongest factor involved: the high percentage of significant nocturnal tornadoes is consistent 

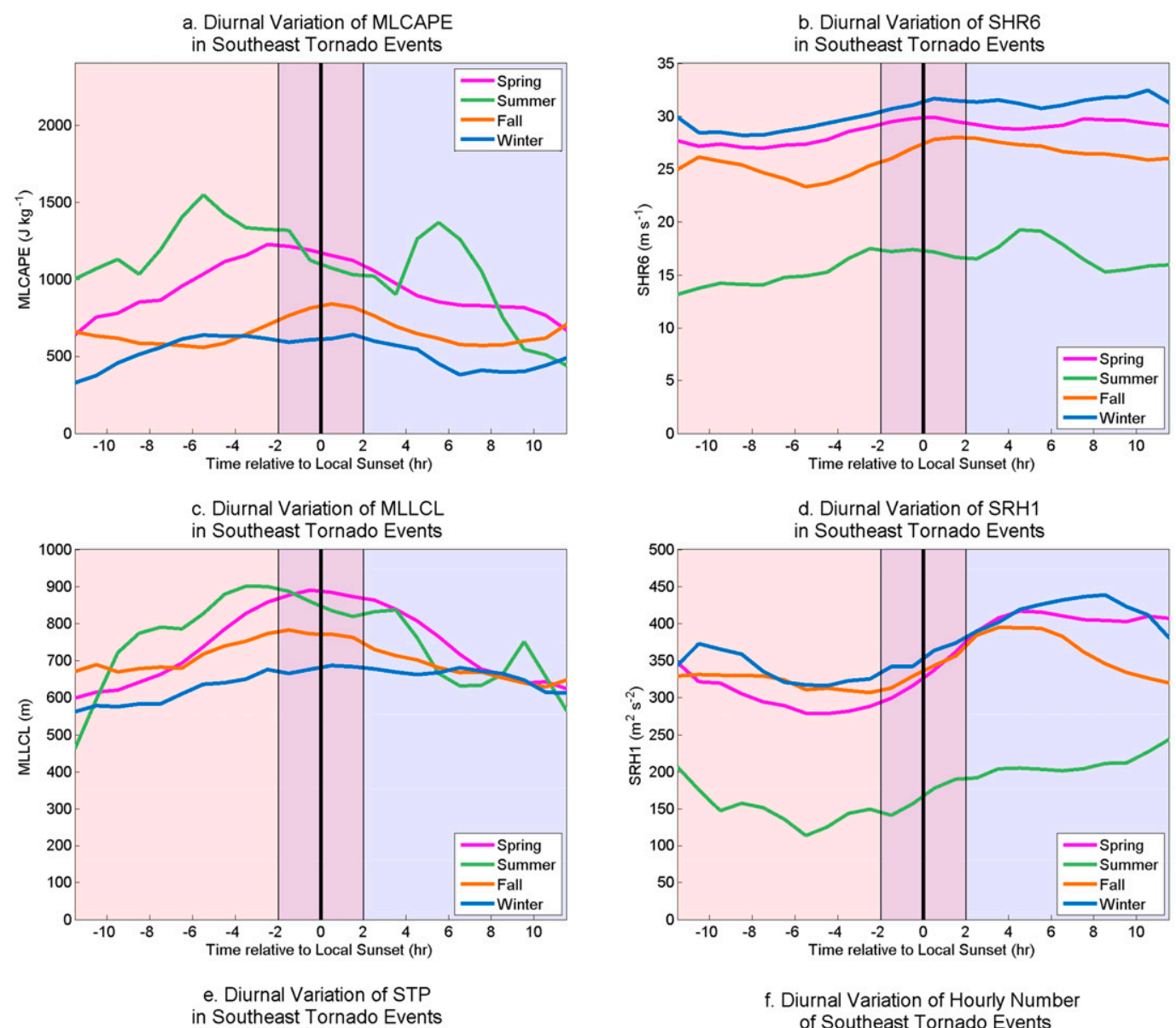

in Southeast Tornado Events
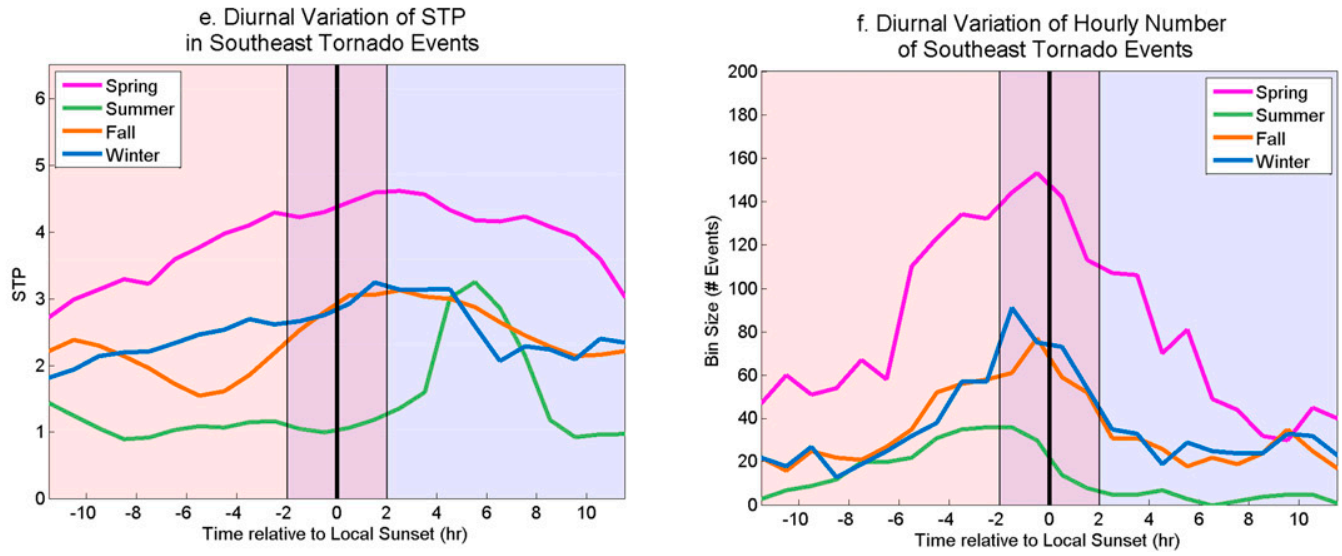

FIG. 4. Diurnal variation of Southeast tornado event (a) MLCAPE, (b) SHR6, (c) MLLCL, (d) SRH1, and (e) STP, with (f) the number of events sorted into each bin. Each season is depicted in a different color. The black vertical line on each plot indicates local sunset, the EET is the shaded area near sunset, and the light red indicates hours presunset while the light blue indicates hours postsunset. Note that $y$ axes are different for all plots. A 5-h moving average is used to smooth the hourly mean data.

with the higher SRH values for tornadoes reported at night (Thompson et al. 2012).

QLCS tornadoes make up $12.4 \%$ of all tornadoes in the CONUS, but that percentage increases to $17.9 \%$ for Southeast tornadoes, and further increases to $23.2 \%$ for HSLC Southeast tornadoes. Nocturnal tornadoes in the Southeast are most common in AL and MS (22.2\% and $19.7 \%$, respectively, of all nocturnal tornadoes in the Southeast), as are winter tornadoes ( $18.5 \%$ and $23.7 \%$, respectively); thus, this Southeast dataset provides an opportunity to study "nontraditional" tornado events outside of the typical 
TABLE 2. Percentage of significant $[(\mathrm{E}) \mathrm{F} 2+]$ tornadoes for the entire dataset of Southeast tornado events and the HSLC subset, further divided by season and time of day.

\begin{tabular}{|c|c|c|c|c|c|c|c|c|}
\hline & All & Spring & Summer & Fall & Winter & Day & EET & Night \\
\hline Southeast (E)F2+ & 21.0 & 24.9 & 7.6 & 13.7 & 24.6 & 17.1 & 20.4 & 26.0 \\
\hline HSLC Southeast (E)F2+ & 19.4 & 19.9 & 10.1 & 14.8 & 23.5 & 16.0 & 18.1 & 24.8 \\
\hline
\end{tabular}

Great Plains springtime early evening RMS tornado paradigm.

\section{b. Warning skill and human impacts}

The Southeast's tornado environments, compared to those of the CONUS as a whole, are shifted toward higher values of SRH1 and SHR6 and lower values of MLCAPE. Given how many forecasting indices and rules of thumb have been built with the traditional Great Plains tornadic environment in mind, how does warning skill measure up in the Southeast?

Two imperfect but commonly used metrics of warning skill are the probability of detection (POD; the percentage of all tornadoes that had a lead time greater than $0 \mathrm{~min}$ ) and the false alarm ratio (FAR; the percentage of all tornado warnings that did not receive an associated report). Generally speaking, unless there is a major paradigm shift in terms of technology or training, a small reduction in FAR results in a relatively large decrease in POD (Brooks 2004). The considerable impact of a missed tornado detection event suggests POD should be given more weight than the more nebulous and difficult-todefine effect of repeated false alarms (Simmons and Sutter 2009, Ripberger et al. 2015), but the combination of the two parameters tells a more detailed story than the POD alone.

For tornadoes in the Southeast, POD is $71.5 \%$ and FAR is $78.6 \%$. In contrast, for the entire CONUS, POD is lower (worse) at $65.6 \%$ and FAR is lower (better) at $75.6 \%$; hence, in the Southeast, both correct (tornadic) and incorrect (nontornadic) storms are being warned at a higher relative rate. In Fig. 5, we break down parameter spaces into bins and calculate POD and FAR for each of these bins (see Anderson-Frey et al. 2016 for details).

It is apparent with a glance at Figs. 5a and $5 \mathrm{c}$ that the parts of the parameter space in which POD is high are also the parts of the parameter space in which FAR is low; that is, warning skill as measured by detection of tornadoes and warning skill as measured by avoidance of false alarms are both enhanced in the same part of the parameter space. The combination of high values of MLCAPE and SHR6 results in more favorable values of both POD and FAR (Figs. 5a,c).

For the second parameter space, however, the area of most favorable FAR is for the combination of high
SRH1 values and relatively high MLLCL, whereas the area of most favorable POD is for high SRH1 values, regardless of MLLCL (Figs. 5b,d). This suggests that the worst warning performance (comparatively speaking) is for the lowest-MLLCL cases, where the environment is nearly saturated. This result is counter to the association between significant tornadoes and low LCL heights found by previous studies (e.g., Craven and Brooks 2004 and Thompson et al. 2012); note, however, that the dataset in Fig. 5 includes many (E)F0-1 (i.e., nonsignificant) tornadoes, whose range of MLLCL heights has been found (Thompson et al. 2012) to overlap considerably with that of nontornadic supercells. When these tornadoes are removed from the events dataset, while the aforementioned POD trends in MLCAPE, SHR6, and SRH1 are maintained, POD no longer decreases with decreasing MLLCL heights; unfortunately, as (E)F-scale ratings cannot be assigned to false-alarm warnings, a similar shift cannot be demonstrated in the FAR data. As it stands, the lack of clear relationship between warning performance and MLLCL heights may be a reflection of the "Goldilocks problem" of baroclinity described by Markowski and Richardson (2009), in which baroclinic enhancement of ambient vorticity may actually be suppressed by particularly extreme (high) values of low-level relative humidity.

Are the deadliest storms being warned? Figure 6a depicts the percentage of each category that resulted in at least one death; this percentage is higher for $(\mathrm{E}) \mathrm{F} 2+$ tornadoes $(16.5 \%)$ than it is for $(\mathrm{E}) \mathrm{F} 0-1$ tornadoes $(0.6 \%)$, and well over half of all $(\mathrm{E}) \mathrm{F} 4+$ tornadoes result in deaths. POD follows a similar pattern (not shown): for (E)F2 + tornadoes, it is $86.9 \%$, whereas for (E)F0-1 tornadoes, it is only $67.6 \%$; the POD for (E)F4+ tornadoes is $100 \%$. Hence, the tornadoes with the lowest probability of detection also tend to be weaker and less likely to be deadly.

The percentage of deadly tornadoes in the Southeast (Fig. 6a) also has a seasonal and time-of-day component, with highest fatality rates during spring and winter as well as during the evening. On average, $2.0 \%$ of tornadoes in the CONUS result in deaths; this number rises to $3.8 \%$ in the Southeast (i.e., $3.8 \%$ of all Southeast tornadoes result in at least one death). Figure $6 \mathrm{~b}$ depicts the fraction of all tornadoes resulting in deaths for 

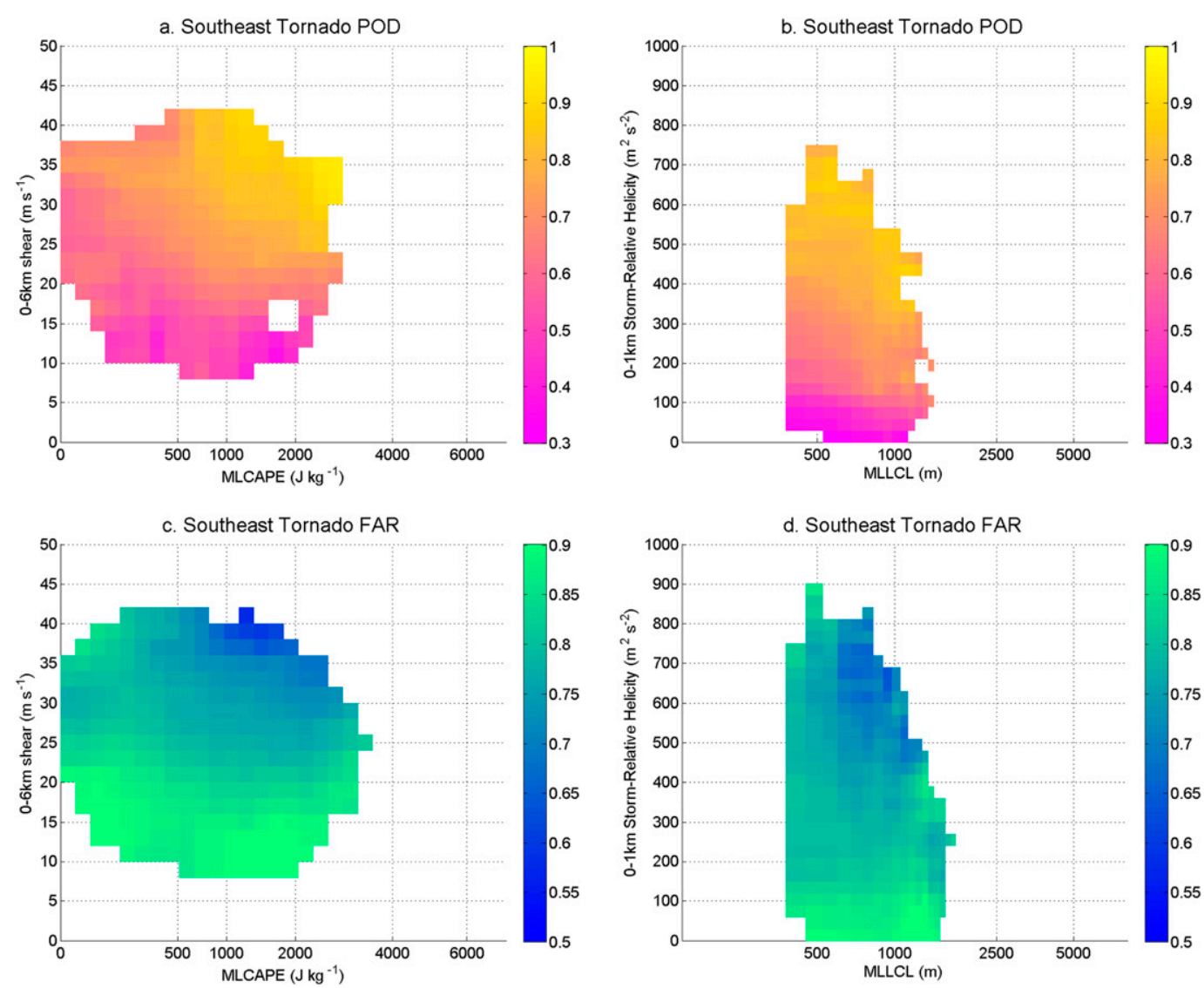

FIG. 5. Binned (top) POD and (bottom) FAR for Southeast tornado reports and warnings for the (a),(c) MLCAPE-SHR6 and (b),(d) MLLCL-SRH1 parameter spaces. Only bins containing at least 10 reports or warnings are shown, and all bins are smoothed using the mean of a $5 \times 5$ bin box surrounding each value.

each hourly bin. Summertime tornadoes have the lowest likelihood of being deadly (no deaths were reported for tornadoes occurring in these seven states during the period of this dataset), followed by tornadoes occurring in fall. Both spring and winter have relatively high fractions of deadly tornadoes, with peaks occurring near local sunset. Fall has a relatively unchanging percentage of deadly tornadoes throughout the day; the jump overnight is likely the result of noise due to the small number of fall events occurring at those times.

The influence of storm mode can also be investigated with the values presented in Table 1, with higher mean percentages of deadly tornadoes in evidence for the RMS subset than for the QLCS subset. For RMS tornadoes, as for the entire dataset, deadly tornadoes occur most frequently for high-(E)F-scale events [18.5\% of (E)F2 + tornadoes, versus $0.5 \%$ of $(\mathrm{E}) \mathrm{F} 0-1$ tornadoes], during the winter $(7.0 \%)$ and spring $(6.6 \%)$, and during the EET $(5.7 \%)$ and overnight $(5.5 \%)$.
Due in part to the low overall percentage of deadly QLCS tornadoes (1.0\%; cf. 5.1\% for RMS tornadoes), and in part to the low percentage of (E)F2+ QLCS tornadoes (10.7\%; cf. $25.1 \%$ for RMS tornadoes), there is no coherent pattern in the percentage of deadly QLCS tornadoes by (E)F scale (Table 1). The percentage of deadly QLCS tornadoes is slightly higher in the fall $(1.6 \%)$ than the winter $(1.1 \%)$ and is higher in the EET $(2.8 \%)$ than either the night $(0.7 \%)$ or the day $(0.4 \%)$.

\section{High-shear low-CAPE tornado events and warnings}

HSLC tornado events have been a focus of recent research since their description as a "key subclass" of severe weather by Schneider et al. (2006) (see also Sherburn and Parker 2014; Sherburn et al. 2016). These events are characterized as having MLCAPE values less than $1000 \mathrm{~J} \mathrm{~kg}^{-1}$ alongside SHR6 values greater than $18 \mathrm{~m} \mathrm{~s}^{-1}(35 \mathrm{kt})$; of the 4133 tornado events and 16429 

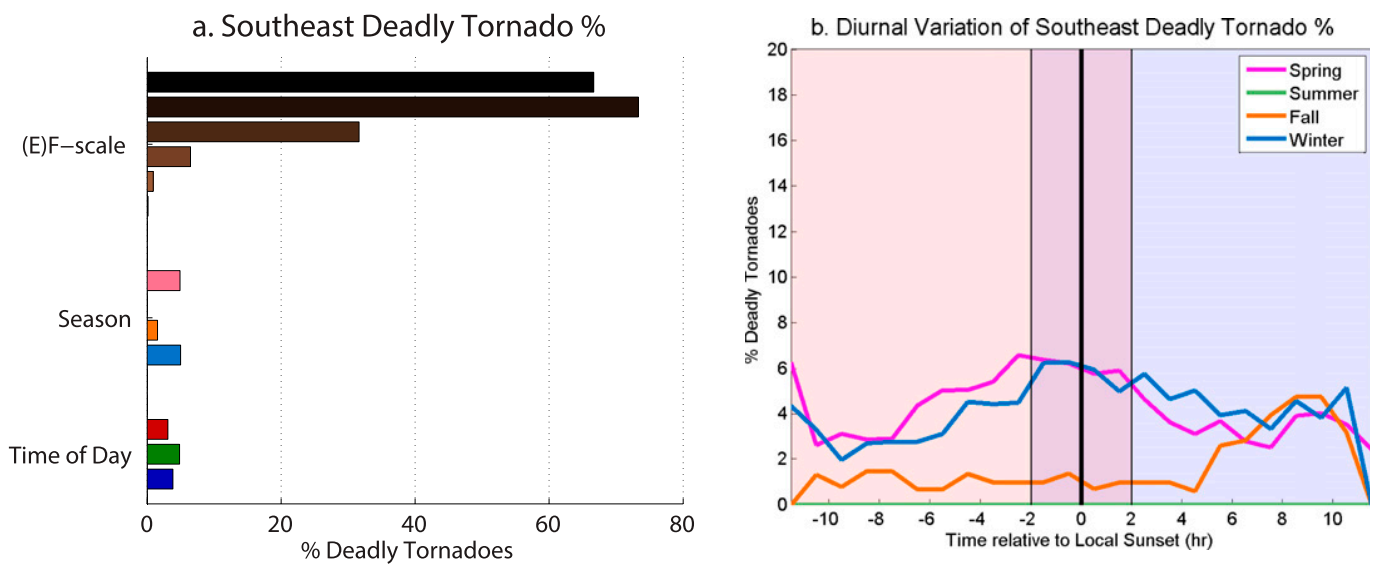

c. Legend

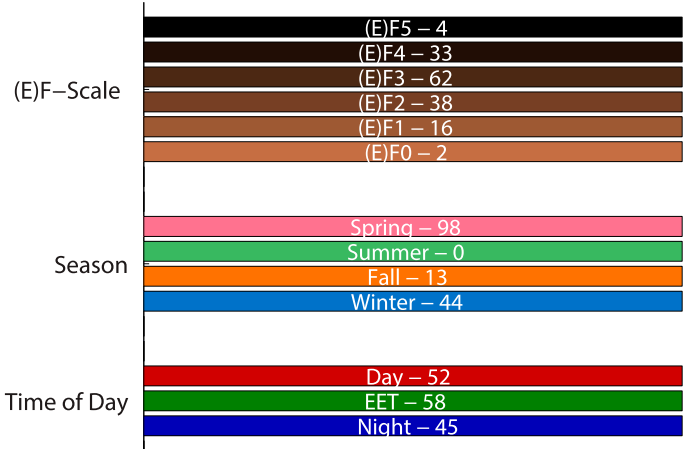

FIG. 6. Deadly tornado information for the Southeast. (a) As in Fig. 3, but for the percentage of deadly tornadoes. (b) As in Fig. 4, but for the percentage of deadly tornadoes in a given hour bin. (c) A color bar legend, along with the number of deadly Southeast tornadoes falling into each category.

tornado warnings in our Southeast dataset, 2509 events $(60.7 \%)$, and 8709 warnings $(53.0 \%)$ match these MLCAPE and SHR6 criteria.

\section{a. Environmental factors}

Environmental parameter values are summarized in Table 3 for all Southeast tornado events and for HSLC tornado events; apart from the obvious differences (the HSLC events have, by definition, higher SHR6 and lower MLCAPE than the full set of Southeast events), HSLC events also tend to have slightly lower MLLCL heights and slightly higher values of SRH1 compared with the full set of Southeast events, with lower STP values overall reflecting the sharp drop in MLCAPE. When compared with all Southeast tornadoes, the MLLCL-SRH1 parameter space (Fig. 7a) shows very little difference between the two categories.

Table 3 also depicts mean environmental parameter values for the RMS and QLCS subsets of the data; for all parameters plotted, RMS tornadoes have, on average, higher (MLCAPE, MLLCL) or comparable (SHR6, SRH1) values when compared with QLCS events for all Southeast tornado events as well as for the HSLC subset. This results in higher mean values of STP for RMS tornadoes than for QLCS tornadoes.
Figure 8 splits the HSLC Southeast events by (E)F scale [noting that there were no (E)F5 tornadoes in this dataset], season, and time of day. Generally speaking, SHR6 (Fig. 8b) and SRH1 (Fig. 8d) increase monotonically with (E)F scale, whereas MLCAPE (Fig. 8a) and MLLCL (Fig. 8c) show very little change; this results in an increase in STP values (Fig. 8e) with (E)F scale.

Seasonally, the thermodynamic parameters (MLCAPE and MLLCL) have nearly the opposite pattern of the kinematic parameters (SHR6 and SRH1), with thermodynamic (kinematic) parameters having their highest (lowest) values in the summer. The seasonal values of STP (Fig. 8e) are lowest for the summer, reflecting its especially low kinematic parameter values.

There are again no prominent patterns in time of day for the parameters examined; MLLCL heights (Fig. 8c) are somewhat lower in the EET and SRH1 (Fig. 8d) is somewhat higher overnight. Overall, STP for these HSLC environments is slightly higher at night than during the EET or the daytime (Fig. 8e), although the overlap of the boxes suggests this finding is not significant.

Table 4 expands this perspective to incorporate the HSLC Southeast events as separated into RMS and QLCS storm modes. For both RMS and QLCS HSLC Southeast events, there is a monotonic increase with 
TABLE 3. As in Table 1, but featuring mean values for all Southeast and Southeast HSLC tornado events.

\begin{tabular}{|c|c|c|c|c|c|c|c|c|c|c|c|c|c|c|c|}
\hline & \multicolumn{3}{|c|}{$\begin{array}{l}\text { MLCAPE } \\
\left(\mathrm{J} \mathrm{kg}^{-1}\right)\end{array}$} & \multicolumn{3}{|c|}{ SHR6 $\left(\mathrm{m} \mathrm{s}^{-1}\right)$} & \multicolumn{3}{|c|}{$\operatorname{MLLCL}(\mathrm{m})$} & \multicolumn{3}{|c|}{ SRH1 $\left(\mathrm{m}^{2} \mathrm{~s}^{-2}\right)$} & \multicolumn{3}{|c|}{ STP } \\
\hline & All & RMS & $\overline{\text { QLCS }}$ & All & RMS & $\overline{\text { QLCS }}$ & All & RMS & QLCS & All & RMS & $\overline{\text { QLCS }}$ & All & RMS & QLCS \\
\hline Southeast tornado events & 869 & 922 & 597 & 27.5 & 28.1 & 28.1 & 744 & 758 & 691 & 326 & 334 & 341 & 3.16 & 3.59 & 1.94 \\
\hline Southeast HSLC tornado events & 447 & 478 & 344 & 29.4 & 29.4 & 29.9 & 728 & 752 & 664 & 356 & 355 & 372 & 1.93 & 2.11 & 1.53 \\
\hline
\end{tabular}

(E)F scale of MLCAPE, SHR6, SRH1, and STP, with MLLCL showing a more complicated relationship once more. For both modes, thermodynamic parameters (MLCAPE and MLLCL) are highest and kinematic parameters (SHR6 and SRH1) are lowest in the summer. For RMS HSLC Southeast tornadoes, STP is moreor-less equally high in the spring (2.21) as in the winter (2.22), whereas for the QLCS storm mode, spring's value (1.70) is more clearly higher than winter's (1.42). Both storm modes have their highest values of MLCAPE during the day, SHR6 and MLLCL in the EET, and SRH1 and STP at night.
The diurnal shift in these parameters is depicted for each season in Fig. 9; once again, note that summer events are considerably less common than the other seasons and will hence appear more noisy (Fig. 9f). Removing the high-CAPE events has resulted in very similar MLCAPE distributions for tornadoes occurring in all seasons (Fig. 9a); in the full Southeast event dataset, springtime tornadoes generally featured higherMLCAPE environments than tornadoes in the other seasons (Fig. 4a). The amplitude of the diurnal signal in MLCAPE also has lessened (Fig. 9a), consistent with nearly saturated environments.
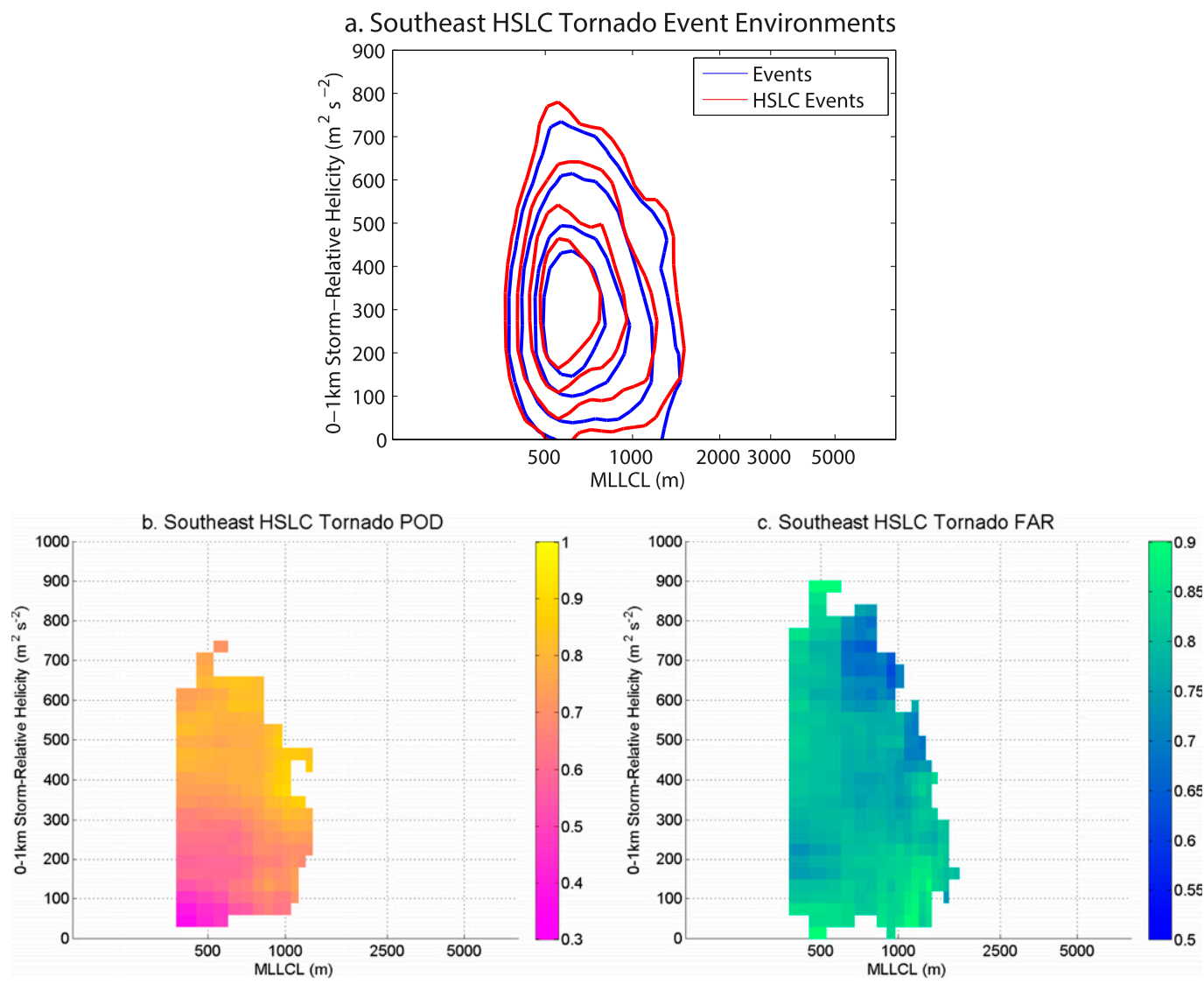

FIG. 7. (a) As in Fig. 2b, but for Southeast tornado events (blue) and Southeast HSLC tornado events (red). (b),(c) As in Figs. 5b and 5d, but for Southeast HSLC tornado events. Note that MLCAPE-SHR6 HSLC plots would simply be subsets of the previously presented data. 
a. Southeast HSLC Tornado Event MLCAPE

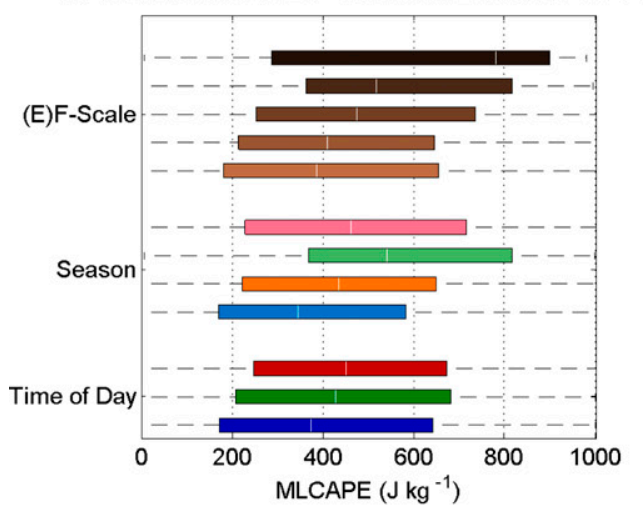

c. Southeast HSLC Tornado Event MLLCL

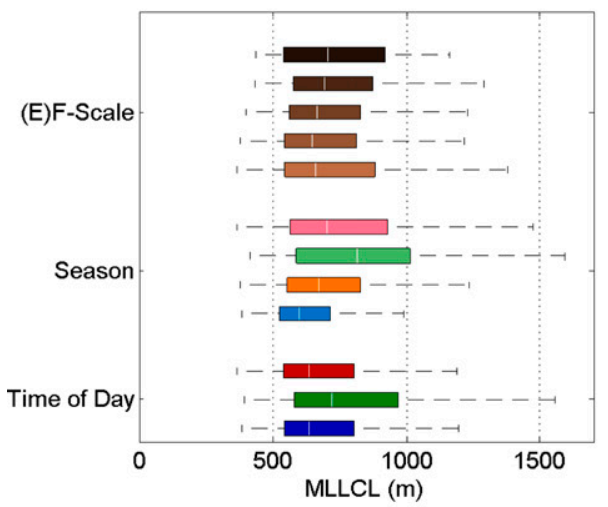

e. Southeast HSLC Tornado Event STP

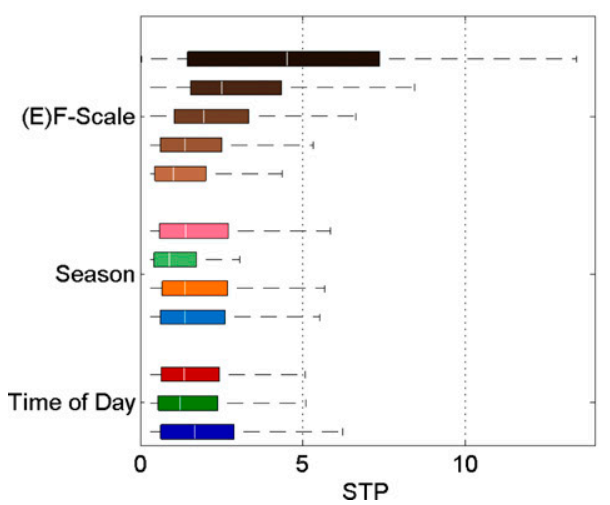

b. Southeast HSLC Tornado Event SHR6

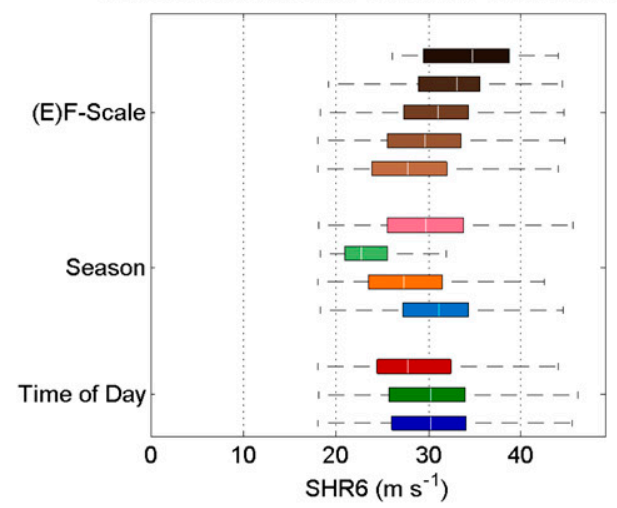

d. Southeast HSLC Tornado Event SRH1

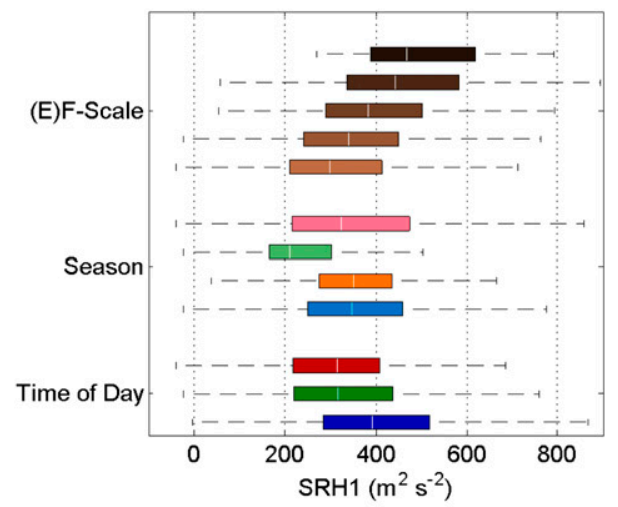

f. Legend

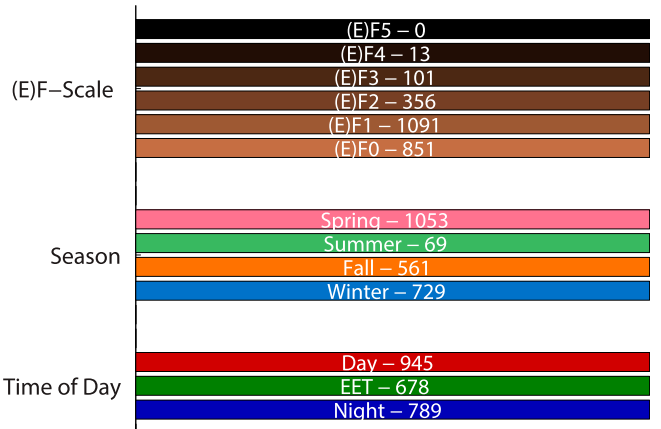

FIG. 8. As in Fig. 3, but for Southeast HSLC tornado events. Note that there are no (E)F5 tornadoes in this data subset. Values in (f) are the number of Southeast HSLC events falling into each category.

While all seasons have similar SHR6 and SRH1 values for tornadoes occurring in the early morning hours, the summer has notably lower values of both throughout most of the day, EET, and night (Figs. 9b,d). Assuming the summer trend is drowned out by the noise of the smaller dataset, the spring is the only season with a pronounced diurnal trend in MLLCL for tornado events (Fig. 9c), with a peak around local sunset. In terms of STP (Fig. 9e), spring sees a slight nocturnal rise in STP commensurate with its nocturnal increase in SRH1 (Fig. 9d).
Of all HSLC tornadoes in the Southeast, 19.4\% (470) are significant $[(\mathrm{E}) \mathrm{F} 2+$; Table 2]; comparing this with $21.0 \%(868)$ significant tornadoes for the Southeast as a whole, it is apparent that the removal of high-MLCAPE events has not dramatically reduced the percentage of significant tornadoes in the dataset, although the absolute number of significant tornadoes has decreased. In this HSLC dataset, winter tornadoes have the highest percentage of significant tornadoes at $23.5 \%$, which is higher even than springtime tornadoes $(19.9 \%)$. 


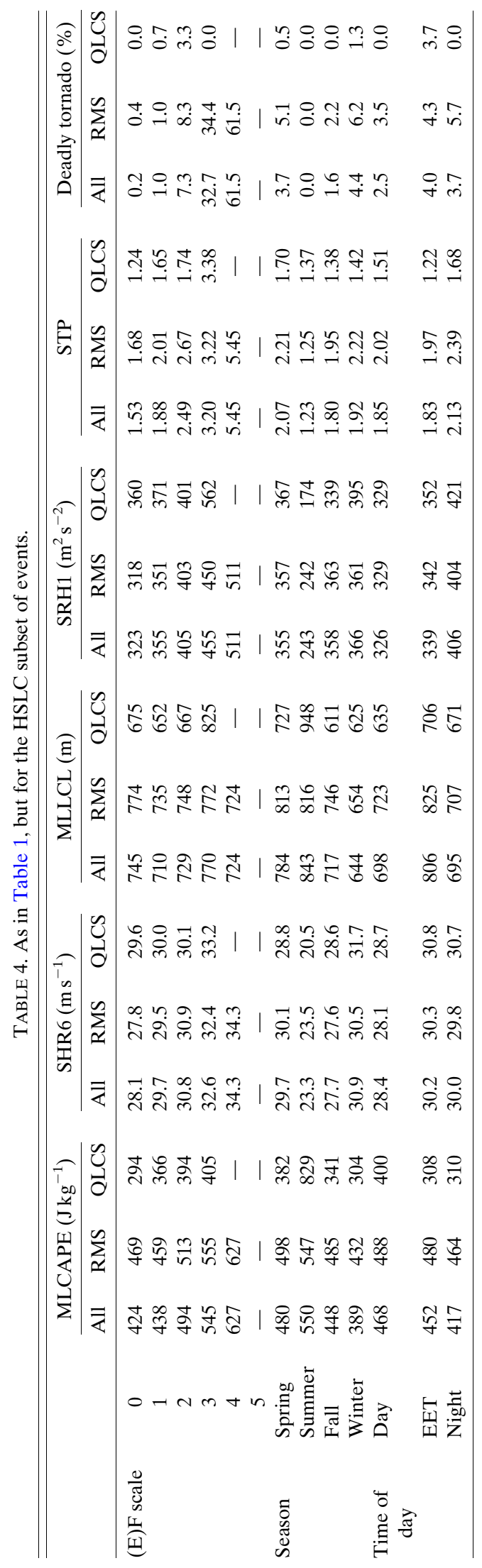

In contrast, only $14.8 \%$ of fall tornadoes and $10.1 \%$ of summer tornadoes are significant. As with the full Southeast tornado dataset, nocturnal tornadoes are more likely to be significant $(24.8 \%)$ than tornadoes occurring during the day $(16.0 \%)$ or the EET $(18.1 \%)$; across all times of day, however, (E)F1 HSLC tornadoes are more common than (E)F0 HSLC tornadoes $(45.2 \%$ and $35.3 \%$, respectively).

\section{b. Warning skill and human impacts}

The POD and FAR plots of Fig. 5 suggest that relatively low-MLCAPE environments are challenging to forecast, whereas relatively high-SHR6 environments are frequently warned correctly (i.e., with high POD and low FAR); these findings match those found in the CONUS as a whole (Brotzge et al. 2013, Anderson-Frey et al. 2016). Does the HSLC subset of the Southeast environment show improvements in these metrics of warning skill compared to the Southeast as a whole? For HSLC environments in the Southeast, POD is $71.8 \%$ (similar to the POD of $71.5 \%$ for all Southeast environments) and FAR is $78.7 \%$ (similar to the FAR of $78.6 \%$ for all Southeast environments); it appears that any worsening of warning skill due to the removal of high-MLCAPE environments may be offset by the removal of more challenging low-SHR6 environments.

Figures $7 b, c$ depict the POD and FAR for the MLLCL-SRH1 parameter spaces of the HSLC subset. The MLLCL-SRH1 parameter space is noisier, especially in terms of FAR (Fig. 7c), but there is still a general tendency for values to be lowest (best) for comparatively high values of both MLLCL and SRH1.

The percentage of deadly tornadoes in the HSLC subset is slightly lower than the Southeast dataset as a whole ( $3.3 \%$ vs $3.8 \%$, respectively), but is still higher than the percentage of deadly tornadoes in the CONUS $(2.0 \%)$. Figure 10a shows a monotonic increase in the percentage of deadly tornadoes with $(\mathrm{E}) \mathrm{F}$ scale; this percentage is slightly higher during the winter and spring than during the fall or the summer (the latter of which had no deadly tornadoes), and EET or nocturnal tornadoes are more likely to be deadly than daytime tornadoes. Figure 10b shows a slight peak in deadly tornadoes around sunset in the spring, with a slight increase later at night in the winter.

\section{Summary}

By making use of a dataset of thousands of tornado events and reports occurring from 2003-17, we build a climatology of tornadoes in the Southeast region of the United States. The environments in which tornadoes occur in the Southeast can be distinguished from those in the rest of the CONUS by their generally lower 

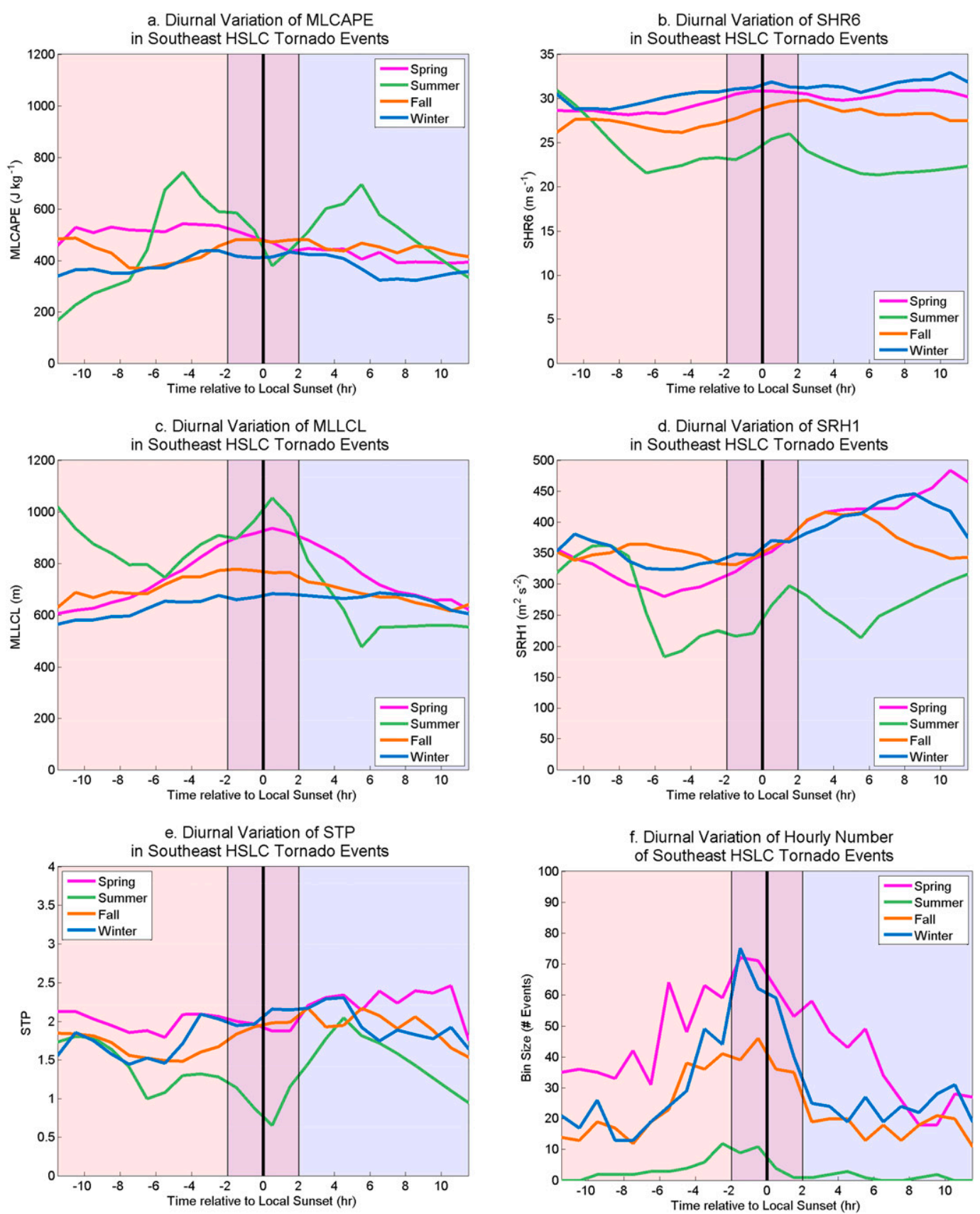

FIG. 9. As in Fig. 4, but for Southeast HSLC tornado events.

MLCAPE and MLLCL values and their generally higher SHR6 and SRH1 values. In terms of STP, the Southeast sees its highest values (i.e., environments generally considered to be most favorable for significant tornadoes) for tornadoes occurring in the springtime and overnight.

The STP for the Southeast as a whole has a coherent diurnal cycle for springtime, in which highest values are around sunset and lowest values are around sunrise; examining only HSLC environments flattens out this cycle by removing much of the diurnal variability in MLCAPE. A relatively high percentage of tornadoes in the Southeast are rated $(\mathrm{E}) \mathrm{F} 2+$, and in both the entire set of southeastern tornadoes and the subset of HSLC southeastern tornadoes, the percentage of (E)F1 tornadoes is greater than that of $(\mathrm{E}) \mathrm{F} 0$ tornadoes. For all times of day, the fraction of tornadoes that are $(\mathrm{E}) \mathrm{F} 0-1$ has increased by about 0.15 when compared with the 1950-76 dataset of Kelly et al. (1978). 
a. Southeast HSLC Deadly Tornado \%

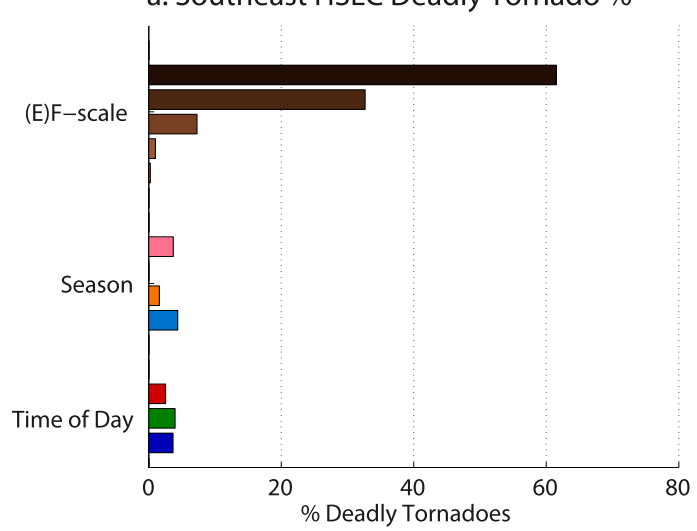

c. Legend

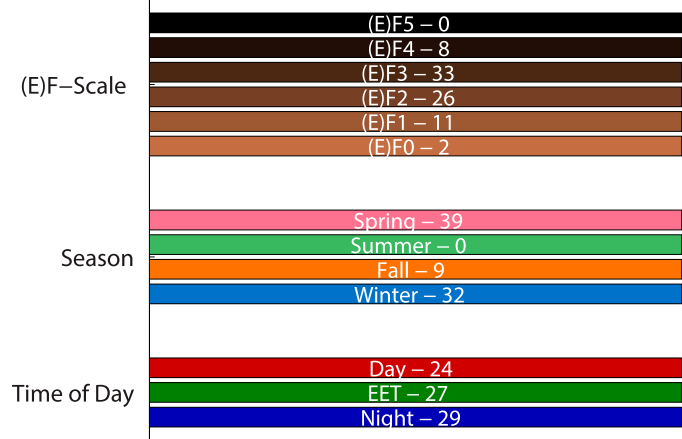

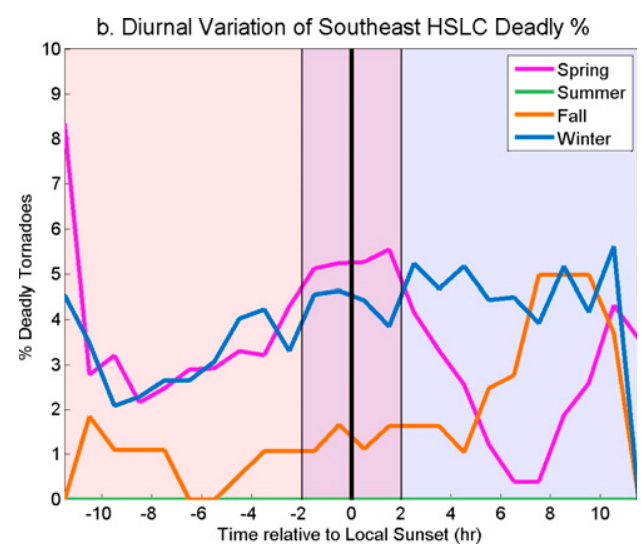

FIG. 10. As in Fig. 6, but for deadly tornado information for HSLC Southeast events. Note that the summer featured no deadly tornado events in the dataset.
For the Southeast, POD is generally better and FAR is generally worse than for the CONUS as a whole. POD and FAR are generally best (highest and lowest, respectively) for the combination of high SHR6 and high MLCAPE; POD and FAR are also generally best for tornadoes occurring in environments with high SRH1, regardless of MLLCL values. These measures remain consistent for the HSLC subset of the southeastern tornado dataset, suggesting that current warning methods are more or less equally effective for this HSLC subset as for the rest of the Southeast cases based on the evaluation metrics of FAR and POD.

The Southeast has nearly double the percentage of deadly tornadoes compared to the CONUS as a whole; spring and winter have a relatively high percentage of deadly tornadoes (with the peak occurring near sunset in both seasons). Over half of all (E)F4+ tornadoes in the Southeast result in deaths, but these tornadoes have a perfect $(100 \%)$ POD. $^{2}$ In general, the tornadoes with the lowest POD also tend to be weaker and less likely to

\footnotetext{
${ }^{2}$ Since no data are available for deaths associated with falsealarm warnings, and since an (E)F-scale rating cannot be assigned to said warnings, a relevant value of FAR cannot be calculated.
}

be deadly. In short, the most dangerous and deadly tornadoes are indeed being well warned, in spite of the many forecasting challenges related to, for example, radar detection in heavily forested areas. Whether the relatively high rates of death associated with these tornadoes could be improved by longer warning lead times, reductions in FAR, or improved communication of warnings and preparedness actions is beyond the scope of the current dataset but bears further investigation.

The next step of this work is to create statistical summaries of these characteristic Southeast tornado environments, using methods such as self-organizing maps to cluster together similar spatial patterns of the variables examined in this work. In contrast with the point-based proximity sounding approach of this work, methods (such as self-organizing maps) that represent fully twodimensional summaries of the near-storm environment will allow for a more nuanced understanding of the spatial patterns involved in these prototypical southeastern tornadic scenarios. This work will also be expanded to provide a deeper understanding of regional, seasonal, and diurnal variations in favorable tornadic environments.

Acknowledgments. The authors are grateful for the assistance from Brenton MacAloney for obtaining the 
verification data, and to three anonymous reviewers and Matt Bunkers for their insightful comments on the manuscript. This work has benefited tremendously from helpful discussions with Harold Brooks, George Young, Martin Tingley, Israel Jirak, Russ Schneider, Richard Grumm, Burkely Gallo, the forecasters at the NWS State College weather office, Steven Weiss, Bill Bunting, Roger Edwards, and Paul Markowski, as well as the mesoscale research group at Penn State University. Anderson-Frey and Richardson's time is supported by a NOAA VORTEX-SE Award NA17OAR4590189.

\section{REFERENCES}

Agee, E., and S. Childs, 2014: Adjustments in tornado counts, F-scale intensity, and path width for assessing significant tornado destruction. J. Appl. Meteor. Climatol., 53, 1494-1505, https://doi.org/10.1175/JAMC-D-13-0235.1.

_- J. Larson, S. Childs, and A. Marmo, 2016: Spatial redistribution of U.S. tornado activity between 1954 and 2013 J. Appl. Meteor. Climatol., 55, 1681-1697, https://doi.org/ 10.1175/JAMC-D-15-0342.1.

Anderson-Frey, A., Y. Richardson, A. Dean, R. Thompson, and B. Smith, 2016: Investigation of near-storm environments for tornado events and warnings. Wea. Forecasting, 31, 1771-1790, https://doi.org/10.1175/WAF-D-16-0046.1.

,,,,---- and -2017 : Self-organizing maps for the investigation of tornadic near-storm environments. Wea. Forecasting, 32, 1467-1475, https://doi.org/10.1175/WAF-D17-0034.1.

,,,$----\frac{-}{-}$, and -2018 : Near-storm environments of outbreak and isolated tornadoes. Wea. Forecasting, 33, 1397 1412, https://doi.org/10.1175/WAF-D-18-0057.1.

Ashley, W., 2007: Spatial and temporal analysis of tornado fatalities in the United States: 1880-2005. Wea. Forecasting, 22, 1214-1228, https://doi.org/10.1175/2007WAF2007004.1.

- A. Krmenec, and R. Schwantes, 2008: Vulnerability due to nocturnal tornadoes. Wea. Forecasting, 23, 795-807, https:// doi.org/10.1175/2008WAF2222132.1.

Benjamin, S., and Coauthors, 2004: An hourly assimilation-forecast cycle: The RUC. Mon. Wea. Rev., 132, 495-518, https://doi.org/ 10.1175/1520-0493(2004)132<0495:AHACTR>2.0.CO;2.

_ - and Coauthors, 2016: A North American hourly assimilation and model forecast cycle: The Rapid Refresh. Mon. Wea. Rev., 144, 1669-1694, https://doi.org/10.1175/MWR-D-15-0242.1.

Boruff, B., J. Easoz, S. Jones, H. Landry, J. Mitchem, and S. Cutter, 2003: Tornado hazards in the United States. Climate Res., 24, 103-117, https://doi.org/10.3354/cr024103.

Bounds, J., and P. Soulé, 1993: Aspects of tornado climatology in Alabama and Florida's Panhandle, 1962-1986. Southeast. Geogr., 33, 172-193, https://doi.org/10.1353/sgo.1993.0006.

Brooks, H., 2004: Tornado-warning performance in the past and future: A perspective from signal detection theory. Bull. Amer. Meteor. Soc., 85, 837-843, https://doi.org/10.1175/ BAMS-85-6-837.

_ C. Doswell III, and M. Kay, 2003: Climatological estimates of local daily tornado probability for the United States. Wea. Forecasting, 18, 626-640, https://doi.org/10.1175/1520-0434(2003) 018<0626:CEOLDT>2.0.CO;2.

Brotzge, J., S. Nelson, R. Thompson, and B. Smith, 2013: Tornado probability of detection and lead time as a function of convective mode and environmental parameters. Wea. Forecasting, 28, 1261-1276, https://doi.org/10.1175/WAF-D12-00119.1.

CDC, 2012: Tornado-related fatalities-Five states, Southeastern United States, April 25-28, 2011. Morbidity Mortality Weekly Rep., 61, 529-533.

Childs, S., and R. Schumacher, 2018: Cold-season tornado risk communication: Case studies from November 2016 to February 2017. Wea. Climate Soc., 10, 419-433, https://doi.org/ 10.1175/WCAS-D-17-0073.1.

Coffer, B., and M. Parker, 2015: Impacts of increasing low-level shear on supercells during the early evening transition. Mon. Wea. Rev., 143, 1945-1969, https://doi.org/10.1175/MWR-D14-00328.1.

- and - 2017: Simulated supercells in nontornadic and tornadic VORTEX2 environments. Mon. Wea. Rev., 145, 149 180, https://doi.org/10.1175/MWR-D-16-0226.1.

Coleman, T., and P. Dixon, 2014: An objective analysis of tornado risk in the United States. Wea. Forecasting, 29, 366-376, https://doi.org/10.1175/WAF-D-13-00057.1.

Craven, J., and H. Brooks, 2004: Baseline climatology of sounding derived parameters associated with deep, moist convection. Natl. Wea. Dig., 28, 13-24.

Davis, J., and M. Parker, 2014: Radar climatology of tornadic and nontornadic vortices in high-shear, low-CAPE environments in the mid-Atlantic and Southeastern United States. Wea. Forecasting, 29, 828-852, https://doi.org/10.1175/WAF-D-13-00127.1.

Edwards, R., A. Dean, R. Thompson, and B. Smith, 2012: Convective modes for significant severe thunderstorms in the contiguous United States. Part III: Tropical cyclone tornadoes. Wea. Forecasting, 27, 1507-1519, https://doi.org/10.1175/ WAF-D-11-00117.1.

Egentowich, J., M. Kaplan, Y.-L. Lin, and A. Riordan, 2000: Mesoscale simulations of dynamical factors discriminating between a tornado outbreak and non-event over the southeast US Part I: 84-48 hour precursors. Meteor. Atmos. Phys., 74, 129-157, https://doi.org/10.1007/s007030070030.

Grazulis, T., 1990: Significant tornadoes. . ..A 107 year perspective. J. Wind Eng. Ind. Aerodyn., 36, 131-151, https://doi.org/ 10.1016/0167-6105(90)90299-R.

Kelly, D., J. Schaefer, R. McNulty, and C. Doswell III, 1978: An augmented tornado climatology. Mon. Wea. Rev., 106, 1172-1183, https://doi.org/10.1175/1520-0493(1978) 106<1172:AATC $>2.0 . \mathrm{CO} ; 2$.

Kincer, J., 1936: Tornado disasters in the southeastern states, April 1936. Mon. Wea. Rev., 64, 168-171, https://doi.org/10.1175/ 1520-0493(1936)64<168:TDITSS >2.0.CO;2.

Knupp, K., and Coauthors, 2014: Meteorological overview of the devastating 27 April 2011 tornado outbreak. Bull. Amer. Meteor. Soc., 95, 1041-1062, https://doi.org/10.1175/BAMS-D-11-00229.1.

Krocak, M., and H. Brooks, 2018: Climatological estimates of hourly tornado probability for the United States. Wea. Forecasting, 33, 59-69, https://doi.org/10.1175/WAF-D-17-0123.1.

Long, J. A., P. C. Stoy, and T. Gerken, 2018: Tornado seasonality in the southeastern United States. Wea. Climate Extremes, 20, 81-91, https://doi.org/10.1016/j.wace.2018.03.002.

Markowski, P., and Y. Richardson, 2009: Tornadogenesis: Our current understanding, forecasting considerations, and questions to guide future research. Atmos. Res., 93, 3-10, https:// doi.org/10.1016/j.atmosres.2008.09.015.

May, O., and A. Bigham, 2012: After the storm: Personal experiences following an EF4 tornado. J. Pediatr. Nurs., 27, 390-393, https://doi.org/10.1016/j.pedn.2012.02.008. 
Ripberger, J., C. Silva, H. Jenkins-Smith, D. Carlson, M. James, and K. Herron, 2015: False alarms and missed events: The impact and origins of perceived inaccuracy in tornado warning systems. Risk Anal., 35, 44-56, https://doi.org/10.1111/risa.12262.

Schaefer, J., D. Kelly, C. Doswell III, J. Galway, R. Williams, R. McNulty, L. Lemon, and B. Lambert, 1980: Tornadoes: When, where, how often: A study of 29 years of tornado data. Weatherwise, 33, 52-59, https://doi.org/10.1080/ 00431672.1980 .9931888 .

Schneider, R., A. Dean, S. Weiss, and P. Bothwell, 2006: Analysis of estimated environments for 2004 and 2005 severe convective storm reports. 23rd Conf. on Severe Local Storms, St. Louis, MO, Amer. Meteor. Soc., 3.5, https://ams.confex.com/ams/ 23SLS/techprogram/paper_115246.htm.

Sherburn, K., and M. Parker, 2014: Climatology and ingredients of significant severe convection in high shear, low CAPE environments. Wea. Forecasting, 29, 854-877, https://doi.org/ 10.1175/WAF-D-13-00041.1.

_, _ _ J. King, and G. Lackmann, 2016: Composite environments of severe and nonsevere high-shear, low-CAPE convective events. Wea. Forecasting, 31, 1899-1927, https:// doi.org/10.1175/WAF-D-16-0086.1.

Sherman-Morris, K., 2013: The public response to hazardous weather events: 25 years of research. Geogr. Compass, 7, 669-685, https://doi.org/10.1111/gec3.12076.

Simmons, K., and D. Sutter, 2009: False alarms, tornado warnings, and tornado casualties. Wea. Climate Soc., 1, 38-53, https:// doi.org/10.1175/2009WCAS1005.1.

Sims, J., and D. Baumann, 1972: The tornado threat: Coping styles of the North and South. Science, 176, 1386-1392, https://doi.org/10.1126/science.176.4042.1386.

Skaggs, R., 1969: Analysis and regionalization of the diurnal distribution of tornadoes in the United States. Mon. Wea.
Rev., 97, 103-115, https://doi.org/10.1175/1520-0493(1969) 097<0103:AAROTD>2.3.CO;2.

Smith, B., R. Thompson, J. Grams, C. Broyles, and H. Brooks, 2012: Convective modes for significant severe thunderstorms in the contiguous United States. Part I: Storm classification and climatology. Wea. Forecasting, 27, 1114-1135, https:// doi.org/10.1175/WAF-D-11-00115.1.

, A. Dean, and P. Marsh, 2015: Diagnosing the conditional probability of tornado damage rating using environmental and radar attributes. Wea. Forecasting, 30, 914-932, https://doi.org/10.1175/WAF-D-14-00122.1.

Sutter, D., and K. Simmons, 2010: Tornado fatalities and mobile homes in the United States. Nat. Hazards, 53, 125-137, https:// doi.org/10.1007/s11069-009-9416-x.

Thompson, R., R. Edwards, J. Hart, K. Elmore, and P. Markowski, 2003: Close proximity soundings within supercell environments obtained from the Rapid Update Cycle. Wea. Forecasting, 18, 1243-1261, https://doi.org/10.1175/1520-0434(2003)018<1243: CPSWSE $>2.0 . \mathrm{CO} ; 2$.

— B. Smith, J. Grams, A. Dean, and C. Broyles, 2012: Convective modes for significant severe thunderstorms in the contiguous United States. Part II: Supercells and QLCS tornado environments. Wea. Forecasting, 27, 1136-1154, https:// doi.org/10.1175/WAF-D-11-00116.1.

$\longrightarrow,-$ A. Dean, and P. Marsh, 2013: Spatial distributions of tornadic near-storm environments by convective mode. Electron. J. Severe Storms Meteor., 8 (5), http://www.ejssm.org/ojs/ index.php/ejssm/article/viewArticle/125.

Wallace, Z., L. Keys-Mathews, and A. Hill, 2015: The role of experience in defining tornado risk perceptions: A case from the 27 April 2011 outbreak in rural Alabama. Southeast. Geogr., 55, 400-416, https://doi.org/10.1353/ sgo.2015.0035. 